\title{
A Method to Estimate the 3D-Time Structure of the Raindrop Size Distribution Using Radar and Disdrometer Data*
}

\author{
MARC SCHLEISS AND JAMES SMITH \\ Civil and Environmental Engineering, Princeton University, Princeton, New Jersey
}

(Manuscript received 19 September 2014, in final form 9 February 2015)

\begin{abstract}
A geostatistical method to quantify the small-scale 3D-time structure of the drop size distribution (DSD) from the ground level up to the melting layer using radar and disdrometer data is presented. First, 3D-time radar reflectivity fields are used to estimate the large-scale properties of a rain event, such as the apparent motion, spatial anisotropy, and temporal innovation. The retrieved quantities are then combined with independent disdrometer time series to estimate the 3D-time variogram of each DSD parameter. A key point in the procedure is the use of a new metric for measuring distances in moving anisotropic rainfall fields. This metric has the property of being invariant with respect to the specific rainfall parameter being considered, that is, it is identical for the radar reflectivity, rain rate, mean drop diameter, drop concentration, or any other weighted moment of the DSD. Evidence is shown of this fact and some illustrations for a stratiform event in southern France and a convective case in the midwestern United States are provided. The proposed framework offers a series of new and interesting applications, including the possibility to compare the spacetime structure of different rain events, to interpolate radar reflectivity fields in space-time and to simulate 3D-time DSD fields at high spatial and temporal resolutions.
\end{abstract}

\section{Introduction}

The study of the space-time variability of drop size distributions (DSDs) during rainfall, from the ground level up to the freezing level, is key to developing a better understanding of the microphysics of rain (Barthes and Mallet 2013). It plays a crucial role in remote sensing and quantitative estimation of precipitation using ground-based or spaceborne weather radars (e.g., Uijlenhoet and Pomeroy 2001; Cifelli et al. 2002; Smith et al. 2007).

Analyzing the space-time variability of the DSD is a very challenging task. Typically, the DSD is measured using disdrometers on the ground. Because disdrometers on the ground do not provide any spatial information, most studies focus on the temporal evolution of the DSD with respect to rainfall type, wind regime,

\footnotetext{
* Supplemental information related to this paper is available at the Journals Online website.
}

Corresponding author address: Marc Schleiss, Civil and Environmental Engineering, Princeton University, E-Quad E316, Olden Street, Princeton, NJ 08540.

E-mail: schleiss.marc@gmail.com and climatology (e.g., Marshall and Palmer 1948; Joss and Gori 1978; Ulbrich 1983; Sempere Torres et al. 1994; Bringi et al. 2003; Kozu et al. 2006; Ulbrich and Atlas 2007). The only way so far to directly estimate the spatial variability of the DSD at the ground level is to deploy large and expensive disdrometer networks (e.g., Tapiador et al. 2010; Jaffrain et al. 2011).

Additional ways of obtaining information about the spatial and temporal variability of the DSD exist but are limited to indirect retrieval techniques. Important insight into the vertical evolution of the DSD can be obtained from the measurements of vertically pointing Doppler profilers (e.g., Peters et al. 2002, 2005; Williams 2002; Yuter and Houze 2003; Yoshikawa et al. 2010). The retrieved DSDs can, however, be affected by large uncertainties and biases due to vertical winds and turbulence, making them difficult to use in quantitative analyses. Similar limitations apply to estimated DSD fields from polarimetric weather radars (e.g., Zhang et al. 2001; Gorgucci et al. 2002; Brandes et al. 2004; Kim et al. 2010).

Using a geostatistical approach, Schleiss et al. (2009) proposed to model the two-dimensional (2D) structure of the DSD using a simple anisotropic variogram model. Their approach is based on Taylor's hypothesis of frozen 
turbulence (Taylor 1938) and consists of transforming DSD time series into spatial profiles along the average direction of advection. The motion and spatial anisotropy used to define the 2D variograms for each event are estimated from independent radar rain-rate maps. Subsequent developments of this technique to intermittent and temporally advected fields can be found in Schleiss et al. (2012). One of the major limitations of these models is the lack of information about the vertical variability of the DSD. To address this issue in a rigorous way, one would need direct 3D-time DSD observations at high spatial and temporal resolutions. Unfortunately, such data are not available so far.

In the meantime, one can look for alternative ways of approximating the space-time structure of the DSD using simple and idealized models based on available data. Of particular interest are techniques for approximating the 3D-time variability using rainfall time series measurements at the ground and large-scale geometric features derived from radar observations. The starting point for such techniques is provided by Taylor's hypothesis of frozen turbulence and subsequent developments (Potvin 1993; Lepioufle et al. 2012).

The goal of this paper is to show that the methodology proposed by Potvin (1993) and Lepioufle et al. (2012) for modeling the 2D-time variability of rain rates and rain amounts can be extended to 3D-time and applied to more fundamental quantities like the raindrop size distribution. More specifically, we propose and test a geostatistical framework for approximating the full 3Dtime variogram of the DSD from the ground level up to the melting layer using disdrometer time series and radar reflectivity data. The focus is on liquid precipitation only and relatively small scales (i.e., a few hundreds of kilometers and a few hours). The proposed approach nicely complements recent work performed within the framework of the Global Precipitation Measurement mission (e.g., Hou et al. 2014; Le and Chandrasekar 2014; Liao et al. 2014; Williams et al. 2014), with the common objective of improving our understanding and modeling capabilities of precipitation microphysics and storm structures. Possible applications include quantitative analyses and comparisons of rainfall structures for different rain events, quantification of errors and uncertainties affecting radar rain-rate estimates, spacetime interpolation of radar data, and the simulation of artificial DSD fields with a prescribed 3D-time structure.

This paper is structured as follows. Section 2 introduces the models and methodology. The numerical methods are described in section 3. Sections 4 and 5 present two case studies for a stratiform event in southern France and a convective event in the midwestern United States. The advantages and limitations of the proposed method are discussed in section 6 . The conclusions are given in section 7 .

\section{Models}

The following section describes the methodology used to model the space-time structure of advected rainfall fields. Readers who do not wish to go through the entire mathematical development can skip to Eq. (23) describing the general link between the space-time variogram of an advected rainfall field and the temporal variogram at a fixed location.

\section{a. Space-time variogram of advected rainfall fields}

Consider an intrinsically stationary, real-valued spatiotemporal random field $[Z(\mathbf{x}, t), \mathbf{x} \in \mathbb{S}, t \in \mathbb{T}]$, defined over a spatial domain $\mathbb{S} \subset \mathbb{R}^{3}$ and a temporal domain $\mathbb{T} \subset \mathbb{R}$, where $\mathbf{x}$ indicates location and $t$ indicates time. In the following, $Z$ can represent any rainfall-related quantity, such as the rain rate, radar reflectivity, or any other weighted moment of the DSD. By definition, its space-time variogram (which describes the structure of $Z$ ) is given by

$$
\gamma_{Z}^{S T}(\mathbf{h}, \tau)=\frac{1}{2} \mathbb{E}\left\{[Z(\mathbf{x}, t)-Z(\mathbf{x}+\mathbf{h}, t+\tau)]^{2}\right\},
$$

where $\mathbf{h} \in \mathbb{R}^{3}$ is a spatial displacement vector, $\tau \in \mathbb{R}$ is a time shift, $\mathbb{E}$ denotes the expectation, and the superscript $S$ indicates space and $T$ indicates time.

Finding an appropriate model for $\gamma_{Z}^{S T}$ is a challenging task in general. Because it is difficult to think about spatial and temporal variations simultaneously, one often focuses on the spatial and temporal restrictions of $\gamma_{Z}^{S T}$ :

$$
\gamma_{Z}^{S}(\mathbf{h})=\gamma_{Z}^{S T}(\mathbf{h}, 0)
$$

and

$$
\gamma_{Z}^{T}(\tau)=\gamma_{Z}^{S T}(0, \tau)
$$

If these were the only quantities that mattered, then a separable model of the form $\gamma_{Z}^{S T}(\mathbf{h}, \tau)=\gamma_{Z}^{S}(\mathbf{h})+\gamma_{Z}^{T}(\tau)$ would suffice. This separable model, however, implies a fully symmetric space-time structure where $\gamma_{Z}^{S T}(\mathbf{h}, \tau)=$ $\gamma_{Z}^{S T}(-\mathbf{h}, \tau)=\gamma_{Z}^{S T}(-\mathbf{h},-\tau)$. As pointed out by Gneiting (2002), such symmetry properties are unlikely to be appropriate for rainfall fields because the rainfall fields exhibit a clear flow direction over time. A more useful approach in this type of situation is to define a Lagrangian reference system moving in the direction of the dominant flow, thus eliminating the overall motion of the storm during the structural analysis:

$$
(\mathbf{x}, t) \mapsto(\mathbf{x}-\mathbf{u} t, t),
$$


where $\mathbf{u}=\left(u_{x}, u_{y}, u_{z}\right)^{\mathrm{T}}$ represents the apparent motion of $Z$, that is, the space-time direction along which the correlation of $Z$ is maximum (i.e., the variability is minimum). Coordinate transformations like the one above form the basis of Taylor's hypothesis of frozen turbulence (Taylor 1938). Specifically, Taylor's hypothesis states that, for small time lags, the temporal variability of $Z$ can be transformed into spatial variability along the direction of advection, and vice versa:

$$
\gamma_{Z}^{S T}(0, \tau) \approx \gamma_{Z}^{S T}(\mathbf{u} \tau, 0)
$$

Such approximations have been shown to hold for temporal lags on the order of $30 \mathrm{~min}$ (Onof et al. 1996; Li et al. 2009), after which the temporal innovation of the rainfall process becomes too important to be ignored. A generalization of this framework can be found in Potvin (1993), who proposed to add an intrinsic velocity $\tilde{u}$ characterizing the internal activity of the rainfall field rather than its pure advection:

$$
(\mathbf{x}, t) \mapsto(\mathbf{x}-\mathbf{u} t, t \tilde{u}) .
$$

A detailed description of this model for 2D-time fields can be found in Lepioufle et al. (2012). It is motivated by the general assumption that the average variability between two points in space and time increases as a function of the "distance" between these points. The problem, of course, is to define an appropriate metric for measuring equivalent distances in space and time. The role of the intrinsic velocity is to normalize the time axis to make it comparable, in terms of variability, with the spatial dimensions. The spacetime metric behind such a coordinate transformation is given by

$$
d(\mathbf{h}, \tau)=\sqrt{\|\mathbf{h}-\mathbf{u} \tau\|^{2}+\left(\frac{\tau}{\alpha}\right)^{2}},
$$

where $\alpha=1 / \tilde{u}>0$ is called the time regularization factor. Assuming that $\mathbf{u}$ and $\alpha$ are known, we can express the space-time variogram of $Z$ as a one-dimensional function of the distance $d(\mathbf{h}, \tau)$ :

$$
\gamma_{Z}^{S T}(\mathbf{h}, \tau)=\gamma_{Z}^{\mathrm{ISO}}[d(\mathbf{h}, \tau)]
$$

where $\gamma_{Z}^{\text {ISO }}: \mathbb{R} \mapsto \mathbb{R}$ is a well-defined isotropic variogram model. In other words, we assume that the space-time variability (i.e., a four-dimensional function) can be approximated using a much simpler, one-dimensional function of distance. The generalization of this approach to anisotropic rainfall fields is straightforward: for a given apparent motion vector $\mathbf{u}=\left(u_{x}, u_{y}, u_{z}\right)^{\mathrm{T}}$ and time regularization factor $\alpha>0$, we define the anisotropic distance function $\delta: \mathbb{R}^{3} \times \mathbb{R} \mapsto \mathbb{R}$ by

$$
\delta(\mathbf{h}, \tau)=\sqrt{\|\mathbf{A}(\mathbf{h}-\mathbf{u} \tau)\|^{2}+\left(\frac{\tau}{\alpha}\right)^{2}},
$$

where $\mathbf{A}$ is a $3 \times 3$ anisotropy matrix used to rotate and stretch the spatial coordinates such that the variability in the moving Lagrangian reference frame remains the same along all directions. Note that the isotropic distance function in Eq. (7) is just a particular case with $\mathbf{A}=\mathbf{I}$ (the identity matrix). Note also that $\mathbf{A}$ can be expressed as a combination of a rotation $\mathbf{R}$ and a scaling matrix $\mathbf{S}$ :

$$
\mathbf{A}=\mathbf{S} \cdot \mathbf{R} .
$$

This is equivalent to defining a centered anisotropy ellipsoid with semimajor axes $a>0, b>0$, and $c>0$ [scaled such that the $\max (a, b, c)=1$ ] and rotation angles $\theta_{x}, \theta_{y}$, and $\theta_{z}$ around the $x, y$, and $z$ axes defining the directions of principal variability and the corresponding anisotropy ratios:

$$
\begin{aligned}
\mathbf{S} & =\left(\begin{array}{ccc}
\frac{1}{a} & 0 & 0 \\
0 & \frac{1}{b} & 0 \\
0 & 0 & \frac{1}{c}
\end{array}\right), \\
\mathbf{R} & =\mathbf{R}_{z}\left(\theta_{z}\right) \cdot \mathbf{R}_{y}\left(\theta_{y}\right) \cdot \mathbf{R}_{x}\left(\theta_{x}\right), \\
\mathbf{R}_{x}(\theta) & =\left(\begin{array}{ccc}
1 & 0 & 0 \\
0 & \cos \theta & -\sin \theta \\
0 & \sin \theta & \cos \theta
\end{array}\right), \\
\mathbf{R}_{y}(\theta) & =\left(\begin{array}{ccc}
\cos \theta & 0 & \sin \theta \\
0 & 1 & 0 \\
-\sin \theta & 0 & \cos \theta
\end{array}\right),
\end{aligned}
$$

and

$$
\mathbf{R}_{z}(\theta)=\left(\begin{array}{ccc}
\cos \theta & -\sin \theta & 0 \\
\sin \theta & \cos \theta & 0 \\
0 & 0 & 1
\end{array}\right)
$$

The space-time variogram of $Z$ as a function of $\delta(\mathbf{h}, \tau)$ is then given by

$$
\gamma_{Z}^{S T}(\mathbf{h}, \tau)=\gamma_{Z}^{\mathrm{ISO}}[\delta(\mathbf{h}, \tau)]
$$

where $\gamma_{Z}^{\mathrm{ISO}}: \mathbb{R} \mapsto \mathbb{R}$ is a well-defined isotropic variogram model. 


\section{b. Mathematical properties of $\gamma_{Z}^{S T}$}

In the following, we derive some basic mathematical properties of $\gamma_{Z}^{S T}$. The first important property concerns the symmetry of the space-time variogram defined in Eq. (16). Because the space-time metric $\delta$ defined in Eq. (9) is symmetric, we have

$\gamma_{Z}^{S T}(-\mathbf{h},-\tau)=\gamma_{Z}^{S T}(\mathbf{h}, \tau), \quad$ for $\quad \forall \mathbf{h} \in \mathbb{R}^{3}$ and $\forall \tau \in \mathbb{R}$.

The space-time variogram is, however, not fully symmetric:

$$
\gamma_{Z}^{S T}(\mathbf{h}, \tau) \neq \gamma_{Z}^{S T}(\mathbf{h},-\tau)
$$

and

$$
\gamma_{Z}^{S T}(\mathbf{h}, \tau) \neq \gamma_{Z}^{S T}(-\mathbf{h}, \tau)
$$

except for the particular case where $\mathbf{u}=0$. Focusing on the semivariance for pure spatial displacements, one has

$$
\gamma_{Z}^{S T}(\mathbf{h}, 0)=\gamma_{Z}^{\mathrm{ISO}}(\|\mathbf{A h}\|)
$$

which means that the spatial variogram does not depend on $\mathbf{u}$ nor on $\alpha$. On the other hand, if we focus on pure temporal variations, we have

$$
\gamma_{Z}^{T}(\tau)=\gamma_{Z}^{S T}(0, \tau)=\gamma_{Z}^{\mathrm{ISO}}(\beta|\tau|)
$$

where $\beta>0$ is a scaling factor given by

$$
\beta=\sqrt{\|\mathbf{A u}\|^{2}+\left(\frac{1}{\alpha}\right)^{2}} .
$$

This leads to an interesting link between the space-time variogram of $Z$ and its temporal variogram:

$$
\begin{aligned}
\gamma_{Z}^{S T}(\mathbf{h}, \tau) & =\gamma_{Z}^{T}\left[\frac{\delta(\mathbf{h}, \tau)}{\beta}\right] \\
& =\gamma_{Z}^{T}\left[\sqrt{\frac{\|\mathbf{A}(\mathbf{h}-\mathbf{u} \tau)\|^{2}+\left(\frac{\tau}{\alpha}\right)^{2}}{\|\mathbf{A u}\|^{2}+\left(\frac{1}{\alpha}\right)^{2}}}\right] .
\end{aligned}
$$

This important result implies that the space-time variogram of $Z$ is fully defined through its temporal restriction $\gamma_{Z}^{T}$, the apparent motion $\mathbf{u}$, spatial anisotropy $\mathbf{A}$, time regularization factor $\alpha$, and associated space-time metric $\delta$. It also means that we can derive the full spacetime variogram of $Z$ using solely time series measurements. This is particularly useful for quantities like the
DSD for which no direct 3D-time measurements are presently available.

Finally, note that by combining Eqs. (20) and (23), one can express the link between the spatial and the temporal variograms of $Z$ :

$$
\gamma_{Z}^{S}(\mathbf{h})=\gamma_{Z}^{T}\left(\frac{\|\mathbf{A} \mathbf{h}\|}{\beta}\right) .
$$

Similarly, the variability along the direction of advection is given by

$$
\gamma_{Z}^{S T}(\mathbf{u} \tau, \tau)=\gamma_{Z}^{T}\left(\frac{|\tau|}{\alpha \beta}\right)
$$

which is a generalization of Taylor's hypothesis of frozen turbulence taking into account the spatial anisotropy and temporal innovation of $Z$.

\section{c. Invariance of motion, anisotropy, and time regularization}

Another way of looking at the space-time variogram proposed in Eq. (16) is to note that $\gamma_{Z}^{S T}$ is fully defined by two major quantities: 1) an isotropic variogram function $\gamma_{Z}^{\text {ISO }}$, specific to $Z$; and 2) an appropriate space-time metric defined by the apparent motion $\mathbf{u}$, spatial anisotropy $\mathbf{A}$, and time regularization factor $\alpha$. For simplicity, the latter can be assumed constant over the course of the event (for a generalization to timedependent quantities the reader is referred to section 6). A key point here is the fact that $\mathbf{u}, \mathbf{A}$, and $\alpha$ can reasonably be assumed invariant, that is, they are identical no matter which rainfall quantity $Z$ we consider (e.g., reflectivity, rain rate, or any weighted moment of the DSD). Experimental evidence supporting this hypothesis is presented in sections 4 and 5. More specifically we show that the sample estimates of $\mathbf{u}, \mathbf{A}$, and $\alpha$ obtained from measurements of the horizontal reflectivity $Z_{\mathrm{h}}$ and differential reflectivity $Z_{\mathrm{dr}}$ (which is almost uncorrelated with $Z_{\mathrm{h}}$ ) are similar. Consequently, we can estimate $\mathbf{u}, \mathbf{A}$, and $\alpha$ for $Z_{\mathrm{h}}$ and use the same values for all weighted moments of the DSD, including the DSD itself. The only quantity that is specific to $Z$ and changes with the considered rainfall quantity is the shape and magnitude of the isotropic variogram model $\gamma_{Z}^{\text {ISO }}$.

\section{d. Space-time variogram of DSD parameters}

The DSD is a statistical description of the microstructure of rainfall. It describes the expected number of drops with equivolume spherical drop diameters between $D$ and $D+d D$ per unit volume $\left(\mathrm{m}^{3}\right)$ of air:

$$
N(D, \mathbf{x}, t)=N_{t}(\mathbf{x}, t) \nu(D, \mathbf{x}, t),
$$


where $N_{t}(\mathbf{x}, t)\left(\mathrm{m}^{-3}\right)$ represents the drop concentration at location $\mathbf{x}$ and time $t$ and $\nu(D, \mathbf{x}, t)\left(\mathrm{mm}^{-1}\right)$ is a probability density function describing the size distribution of the drop diameters at location $\mathbf{x}$ and time $t$. In this paper, we consider the most widely used model for $\nu$, which is a Gamma distribution (Ulbrich 1983; Willis 1984 ) with shape parameter $\mu(\mathbf{x}, t)>-1$ (unitless) and rate parameter $\Lambda(\mathbf{x}, t)>0\left(\mathrm{~mm}^{-1}\right)$ :

$$
N(D, \mathbf{x}, t)=N_{t}(\mathbf{x}, t) D^{\mu(\mathbf{x}, t)} e^{-\Lambda(\mathbf{x}, t) D} .
$$

In general, $\mu, \Lambda$, and $N_{t}$ are fitted to experimental DSD spectra using maximum likelihood or the method of moments. Further simplifications occur if one assumes that $\Lambda$ is a smooth, deterministic function of $\mu$ (e.g., Zhang et al. 2003; Seifert 2005; Smith et al. 2009). In this way, we can ignore $\Lambda$ and focus on the two major parameters $\mu$ and $N_{t}$. To avoid the tedious task of modeling cross variograms, we further assume that $\mu$ and $N_{t}$ are uncorrelated, even though in reality these quantities usually exhibit a slight negative correlation. This is just a simplification designed to streamline the structural analysis and is not critical with respect to the proposed approach.

Using the parameterization described above, the DSD reduces to a bivariate random field $\left(\mu, N_{t}\right)$ whose 3D-time structure is fully defined by the two space-time variograms $\gamma_{\mu}^{S T}$ and $\gamma_{N_{t}}^{S T}$ :

$$
\gamma_{\mu}^{S T}(\mathbf{h}, \tau)=\frac{1}{2} \mathbb{E}\left\{[\mu(\mathbf{x}, t)-\mu(\mathbf{x}+\mathbf{h}, t+\tau)]^{2}\right\}
$$

and

$\gamma_{N_{t}}^{S T}(\mathbf{h}, \tau)=\frac{1}{2} \mathbb{E}\left\{\left[N_{t}(\mathbf{x}, t)-N_{t}(\mathbf{x}+\mathbf{h}, t+\tau)\right]^{2}\right\}$.

Assuming that $\mathbf{u}, \mathbf{A}$, and $\alpha$ are known and identical for all DSD-related quantities, we can use Eq. (23) to express the space-time variogram of each DSD parameter as a function of its temporal variogram:

$$
\gamma_{\mu}^{S T}(\mathbf{h}, \tau)=\gamma_{\mu}^{T}\left[\frac{\delta(\mathbf{h}, \tau)}{\beta}\right]
$$

and

$$
\gamma_{N_{t}}^{S T}(\mathbf{h}, \tau)=\gamma_{N_{t}}^{T}\left[\frac{\delta(\mathbf{h}, \tau)}{\beta}\right]
$$

where $\gamma_{\mu}^{T}$ and $\gamma_{N_{t}}^{T}$ represent the temporal variograms of $\mu$ and $N_{t}$ and $\delta$ is the non-Euclidean anisotropic space-time metric defined in Eq. (9). This also defines the method for retrieving the 3D-time structure of the DSD parameters from disdrometer time series measurements:
1) estimate $\mathbf{u}, \mathbf{A}$, and $\alpha$ using 3D-time radar reflectivity data in the vicinity of the disdrometers;

2) estimate $\gamma_{\mu}^{T}$ and $\gamma_{N_{t}}^{T}$ using the DSD time series collected by the disdrometer(s); and

3) derive $\gamma_{\mu}^{S T}$ and $\gamma_{N_{t}}^{S T}$ using Eqs. (30) and (31).

\section{Numerical methods}

This section describes the numerical methods used to estimate the apparent motion $\mathbf{u}$, anisotropy matrix A, and time regularization factor $\alpha$ from 3D-time radar observations. For simplicity, the procedure is explained for the radar reflectivity field $Z(\mathrm{~dB} Z)$ only. The same methods can, however, be applied to the horizontal/vertical reflectivity fields $Z_{\mathrm{h} \mid \mathrm{v}}$, differential reflectivity $Z_{\mathrm{dr}}$, or any other weighted moment of the DSD. Note that $\mathbf{u}, \mathbf{A}$, and $\alpha$ can be estimated using different methods. In the following, we provide a method that is based on the experimental space-time variogram of $Z$ :

$\hat{\gamma}_{Z}^{S T}(\mathbf{h}, \tau)=\frac{1}{2\left|S_{\mathbf{h}}^{\tau}\right|} \sum_{(i, j) \in S_{\mathbf{h}}^{\tau}}\left[Z\left(\mathbf{x}_{i}, \tau_{i}\right)-Z\left(\mathbf{y}_{j}, \tau_{j}\right)\right]^{2}$

and

$$
S_{\mathbf{h}}^{\tau}=\left[(i, j) \mid \mathbf{x}_{i}-\mathbf{y}_{j} \approx \mathbf{h} \quad \text { and } \quad \tau_{i}-\tau_{j} \approx \tau\right],
$$

where $\left|S_{\mathbf{h}}^{\tau}\right|$ denotes the number of elements in $S_{\mathbf{h}}^{\tau}$, that is, the number of samples separated approximately by $\mathbf{h}$ in space and $\tau$ in time (up to a given tolerance chosen by the user).

Experimental space-time variograms are fourdimensional functions and therefore rather difficult to visualize (see Fig. 3, described in greater detail below, for an example). A good way to look at them is to analyze the semivariance along different planes of variation. For example, the three spatial planes of variation $x-y, x-z$, and $y-z$ can be used to estimate the anisotropy of the field. The three space-time planes $x-$ $t, y-t$, and $z-t$ can be used to estimate the apparent motion of the field. Finally, the time regularization is estimated by comparing the temporal and spatial variability.

\section{a. Apparent motion}

The apparent motion $\mathbf{u}=\left(u_{x}, u_{y}, u_{z}\right)^{T}$ is defined as the direction of minimum variability (i.e., maximum correlation) along each individual space-time plane $x$ $t, y-t$, and $z-t$. It can be estimated using one of the various techniques proposed in the literature (e.g., Crane 1979; Dixon and Wiener 1993; Mecklenburg et al. 2000; Bowler et al. 2004) or directly from the 
experimental space-time variogram. The latter has the advantage of being very intuitive and fast. The procedure is similar along each axis and will only be explained in detail for $u_{x}$

For a given time lag $\tau>0$, we use the experimental variogram $\hat{\gamma}^{S T}$ to determine the spatial displacement $\mathbf{h}_{x}^{\min }(\tau)$ that minimizes the semivariance along the $x$ axis:

$$
\mathbf{h}_{x}^{\min }(\tau)=\underset{\mathbf{h}_{x}=\left(h_{x}, 0,0\right)^{T}}{\operatorname{argmin}}\left[\hat{\gamma}_{Z}^{S T}\left(\mathbf{h}_{x}, \tau\right)\right] .
$$

Assuming that $\mathbf{h}_{x}^{\min }(\tau)=\left[h_{x}^{\min }(\tau), 0,0\right]^{T}$, we can estimate the apparent velocity along the $x$ axis by

$$
\hat{u}_{x}(\tau)=\frac{h_{x}^{\min }(\tau)}{\tau} .
$$

Because the estimated velocities might depend on the time lag $\tau$, we define the apparent velocity along $x$ as the average over all available time lags up to about $30 \mathrm{~min}$ :

$$
\hat{u}_{x}=\operatorname{mean}_{0<|\tau|<30 \min }\left[\hat{u}_{x}(\tau)\right] .
$$

The upper limit on the time lag is necessary to limit the bias due to temporal innovation and nonhomogeneous motion fields that may alter the direction of minimum variability.

\section{b. Spatial anisotropy}

The anisotropy matrix $\mathbf{A}$ is estimated by fitting ellipsoids to selected semivariance contours. More specifically, for a given semivariance $s>0$ and a small tolerance level $\varepsilon>0$, we define the set $H_{s}$ of spatial displacements:

$$
H_{s}=\left[\mathbf{h} \in \mathbb{R}^{3}|| \hat{\gamma}_{Z}^{S T}(\mathbf{h}, 0)-s \mid \leq \varepsilon\right] .
$$

If $Z$ exhibits geometric anisotropy, $H_{s}$ represents the surface of an ellipsoid with scaling matrix $\hat{\mathbf{Q}}_{s}$ and rotation matrix $\hat{\mathbf{R}}_{s}$ defined through the following optimization problem:

$$
\sum_{\mathbf{h} \in H_{s}}\left(\left\|\mathbf{Q}_{s} \mathbf{R}_{s} \mathbf{h}\right\|-1\right)^{2}=\min !
$$

The corresponding anisotropy matrix is then given by

$$
\hat{\mathbf{A}}_{s}=\frac{\hat{\mathbf{Q}}_{s}}{\max \left(\hat{\mathbf{Q}}_{s}\right)} \hat{\mathbf{R}}_{s},
$$

where $\hat{\mathbf{Q}}_{s}$ and $\hat{\mathbf{R}}_{s}$ are the solutions of the minimization problem in Eq. (38). For more robustness, the procedure is repeated for different values of $s$ and the average anisotropy parameters are chosen.

\section{c. Time regularization}

The time regularization factor $\alpha$ is estimated based on the previous estimates $\hat{\mathbf{u}}$ and $\hat{\mathbf{A}}$. It is obtained by considering the relation between the spatial and temporal variograms given in Eq. (24):

$$
\hat{\alpha}=\underset{\alpha>0}{\operatorname{argmin}}\left\{\sum_{\mathbf{h} \in \mathbb{R}^{3}}\left[\hat{\gamma}_{Z}^{S}(\mathbf{h})-\hat{\gamma}_{Z}^{T}\left(\frac{\|\hat{\mathbf{A}} \mathbf{h}\|}{\hat{\beta}}\right)\right]^{2}\right\}
$$

and

$$
\hat{\beta}=\sqrt{\|\hat{\mathbf{A}} \hat{\mathbf{u}}\|^{2}+\left(\frac{1}{\alpha}\right)^{2}},
$$

which can be solved numerically using iterative nonlinear least squares. Note that before the optimization, a valid variogram model must be fitted on $\hat{\gamma}_{Z}^{T}$ in Eq. (40) to compute the semivariance associated with any given time lag. The choice of the variogram model is not critical as long as it adequately approximates the sample variogram.

This ends the section about the numerical methods for estimating the apparent motion, anisotropy, and time regularization factor from 3D-time radar reflectivity data. The goal of the next two sections is to illustrate the procedure for two different rain events in southern France and the midwestern United States.

\section{Case study 1: Stratiform event}

We consider a stratiform rainfall event on 26 October 2012 in the region of Ardèche, southern France. The precipitation is associated with a cold front moving from southwest to northeast bringing moist air from the Mediterranean to the southern part of France and the Alps. The event was simultaneously observed by a dual-polarization X-band weather radar and seven optical disdrometers of type Parsivel (Löffler-Mang and Joss 2000) deployed over an area of approximately $5 \times 5 \mathrm{~km}^{2}$ and part of the Hydrological Cycle in Mediterranean Experiment (HyMeX; Ducrocq et al. 2014). For homogeneity and stationarity reasons, the analyses focus on a small domain of size $50 \mathrm{~km} \times$ $50 \mathrm{~km} \times 2 \mathrm{~km}$ and a short time interval from 0130 to 0500 UTC during which the rainfall field was moving from southwest to northeast with approximately constant velocity. Animation 1 (available as supplemental material at the Journals Online website: http://dx.doi. org/10.1175/JHM-D-14-0182.s1) shows the temporal evolution of the radar reflectivity field (projected to the ground level) during this period. Figure 1 shows two snapshots of the (projected) radar reflectivity field at 

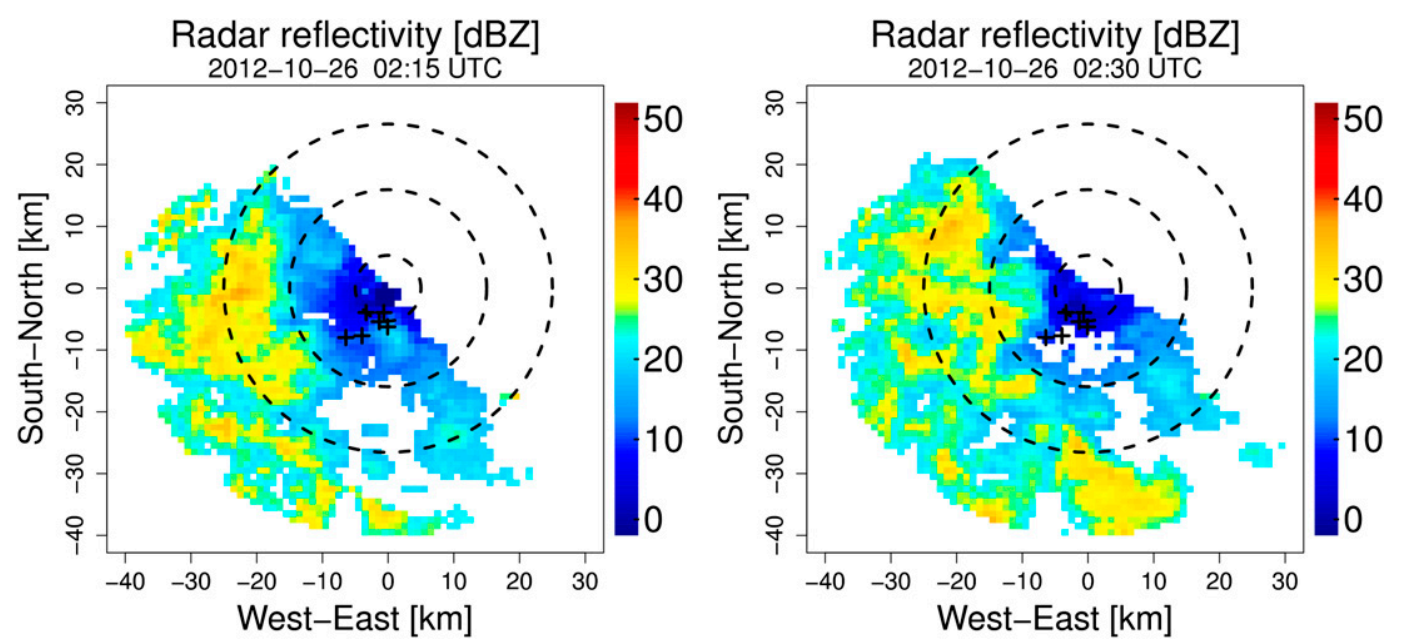

FIG. 1. Radar reflectivity maps (dBZ; corrected for attenuation) for event 1 (stratiform) at (left) 0215 and (right) 0230 UTC, resampled over a Cartesian grid with $1 \times 1 \mathrm{~km}^{2}$ and 5-min resolution and projected to the ground level. The radar is located at the origin $(0,0)$ of the coordinate system. The concentric circles denote the 5-, 15-, and 25-km ranges. The locations of the Parsivel disdrometers are marked by black crosses.

0215 and 0230 UTC together with the position of the Parsivel disdrometers in the area. An aerial view of the studied domain together with the location of the sensors is provided in Fig. 2.

The case study consists of two main parts. First, the 3D-time radar reflectivity data are used to estimate the large-scale features, that is, the apparent motion, anisotropy, and time regularization factor. Based on the obtained values, we construct a theoretical 3D-time variogram model for $Z_{\mathrm{h}}$ and $Z_{\mathrm{dr}}$ using Eq. (23) and verify that the model is in agreement with the experimental space-time variograms. In the second part, we use the collected disdrometer data to estimate the temporal variograms of the DSD parameters $\mu$ and $N_{t}$ and derive a 3D-time variogram model for each of the DSD parameters using the technique described at the end of section $2 \mathrm{~d}$.

\section{a. Space-time variogram of $Z_{\mathrm{h}}$ and $Z_{\mathrm{dr}}$}

The radar data used to derive the space-time variograms of $Z_{\mathrm{h}}$ and $Z_{\mathrm{dr}}$ consist of a large collection of PPI and RHI scans of horizontal and vertical reflectivity $(\mathrm{dB} Z)$ at different elevation angles with a maximum range of $30 \mathrm{~km}$ and a range resolution of about $40 \mathrm{~m}$. All data are corrected for attenuation, ground clutter, and beam blocking. A threshold of $0 \mathrm{~dB} Z$ corresponding to

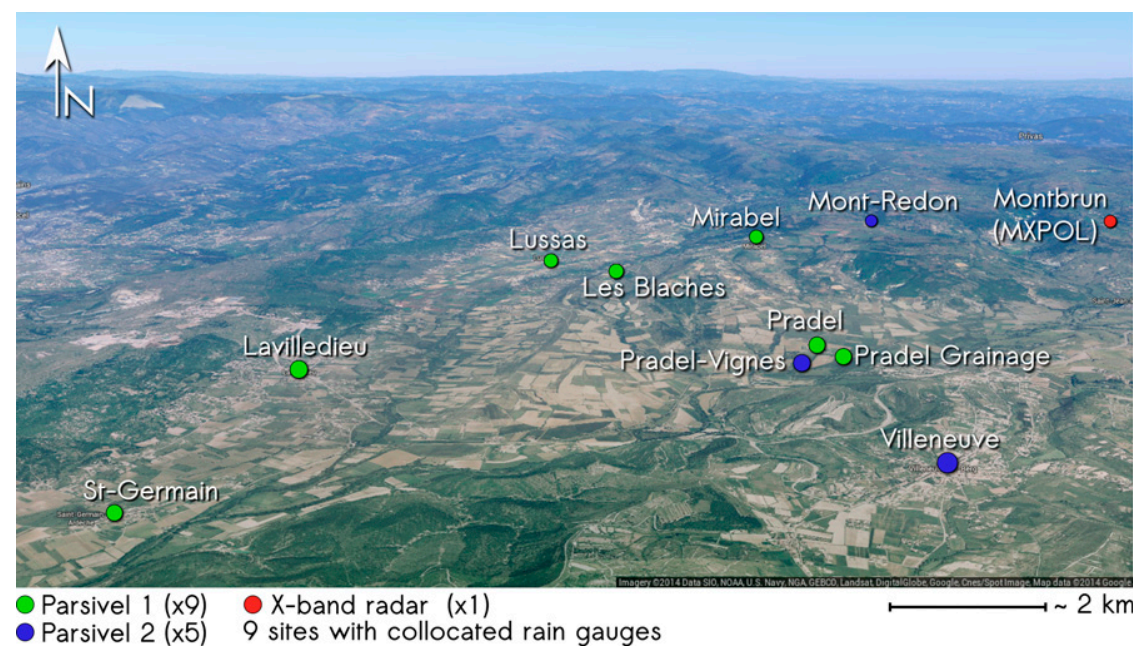

FIG. 2. Aerial view of the studied region in Ardèche, France, during the HyMeX campaign. The Parsivel disdrometers are denoted by green dots, the X-band radar is denoted by a red dot. 

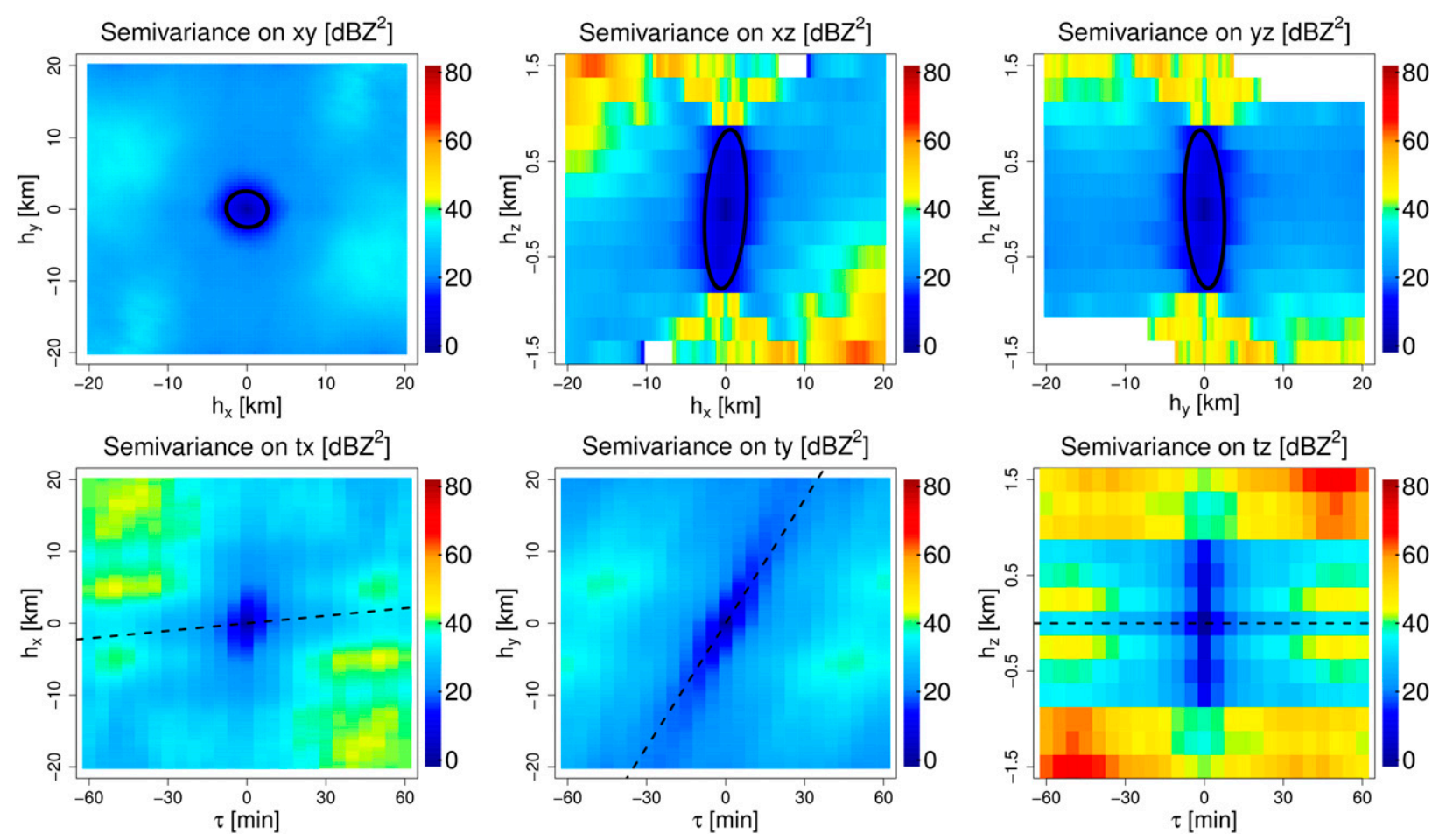

FIG. 3. Experimental space-time variogram of $Z_{\mathrm{h}}$ for event 1 (stratiform) on six orthogonal planes of variation. The class widths are $0.5 \mathrm{~km}$ for $h_{x}$ and $h_{y}, 0.25 \mathrm{~km}$ for $h_{z}$, and $5.0 \mathrm{~min}$ for $\tau$. The min number of samples per class is 100 . The ellipses represent the fitted anisotropy ellipsoid for a fixed value of semivariance. The dotted lines represent the fitted directions of min space-time variability along each axis.

about $0.05 \mathrm{~mm} \mathrm{~h}^{-1}$ is used to distinguish between dry and rainy areas. To ensure only liquid precipitation is considered, the authors discarded all measurements above $2 \mathrm{~km}$ MSL and/or with a copolar cross correlation $\rho_{\text {hv }}$ of less than 0.98 . The exact height of the melting layer varies in space and time and is difficult to determine precisely, but the analysis of the radar data and local temperature data for the region suggests that it remains above $2 \mathrm{~km}$ during the entire event. Before analysis, the selected data were resampled over a Cartesian grid with $0.5-\mathrm{km}$ horizontal, $0.25-\mathrm{km}$ vertical, and 5 -min temporal resolution and were used to compute the experimental space-time variogram of $Z_{\mathrm{h}}$ and $Z_{\mathrm{dr}}$ according to the procedure described in section 3. Given the resolution of the radar data, the variograms were computed using space-time classes of size $0.5 \mathrm{~km} \times$ $0.5 \mathrm{~km} \times 0.25 \mathrm{~km} \times 5 \mathrm{~min}$. To limit the computational cost, the spatial displacements were limited to a maximum of $20 \mathrm{~km}$ on the horizontal axes, $1.5 \mathrm{~km}$ on the vertical axis, and $60 \mathrm{~min}$ in time. The average number of samples per space-time class is on the order of 10000 . Because of the radar scanning strategy, however, some space-time displacements are more common than others. To limit sampling issues, all space-time classes with fewer than 100 samples were discarded during the analysis.

Figures 3 and 4 show the obtained experimental space-time variograms of $Z_{\mathrm{h}}$ and $Z_{\mathrm{dr}}$. The complete variograms have four dimensions and cannot be displayed properly. It is nevertheless possible to get a good idea of their shape by looking at the semivariance values along the six different orthogonal planes $x-y, x-z, y-z$, $x-t, y-t$, and $z-t$. Using the numerical methods defined in section 3, we can estimate $\mathbf{u}, \mathbf{A}$, and $\alpha$ for both $Z_{\mathrm{h}}$ and $Z_{\mathrm{dr}}$ (see Table 1 for the fitted values). One can see that the two space-time variograms are very similar in shape. This is consistent with our assumption that $\mathbf{u}, \mathbf{A}$, and $\alpha$ are invariant characteristics of a rainfall field that are identical for all moments of the DSD.

Because this is a stratiform event, the semivariance values of $Z_{\mathrm{h}}$ and $Z_{\mathrm{dr}}$ are relatively low. The fields also exhibit little anisotropy along the horizontal direction (i.e., along the $x-y$ plane), which is usually the case when there are no convective cells. There is, however, some slight anisotropy along $x-z$ and $y-z$. By focusing on Fig. 3 (bottom) and Fig. 4 (bottom), one can see that $Z_{\mathrm{h}}$ and $Z_{\mathrm{dr}}$ exhibit a clear direction of minimum variability along the $y-t$ plane, consistent with the strong motion 

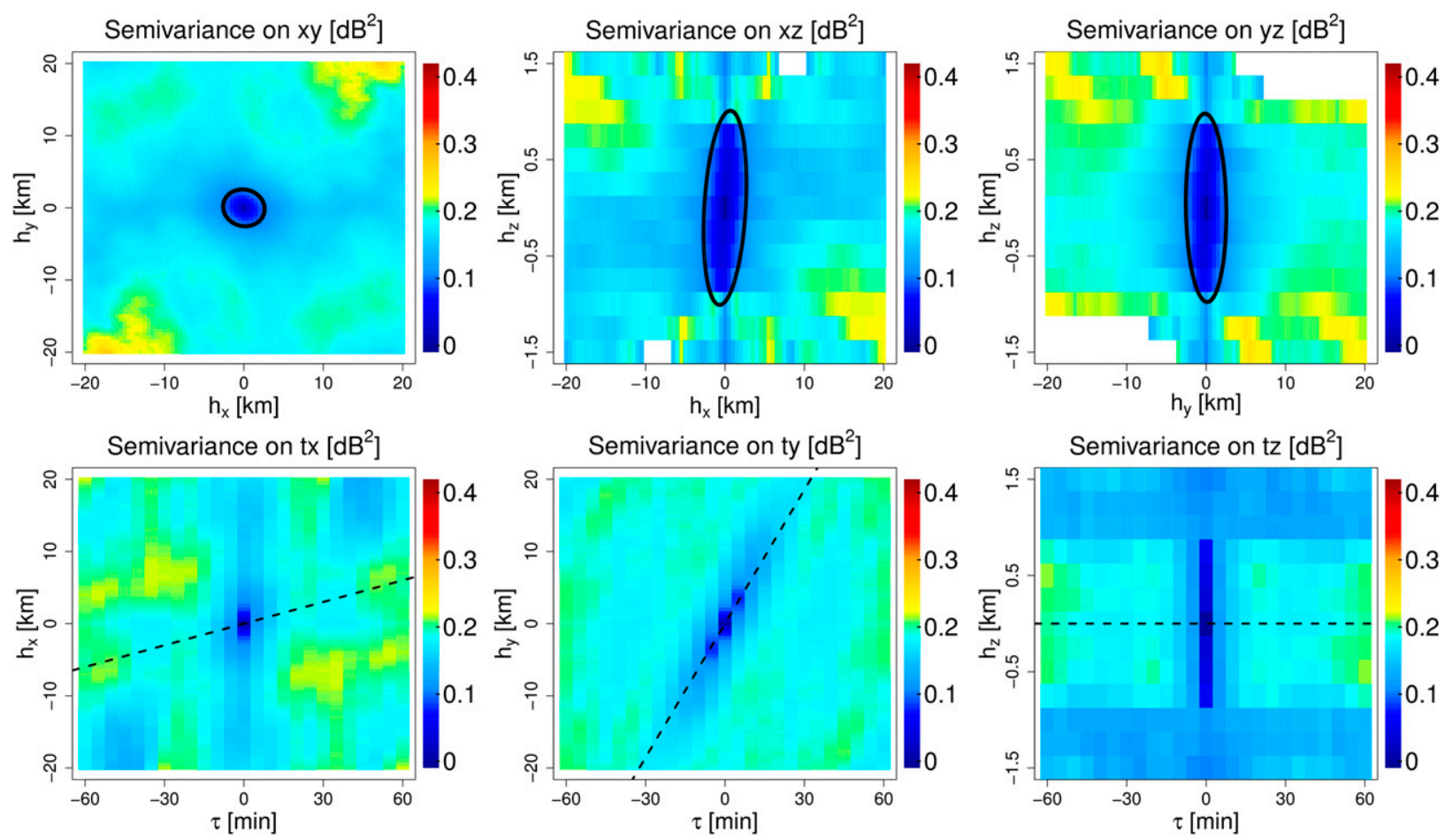

FIG. 4. Experimental space-time variogram of $Z_{\mathrm{dr}}$ for event 1 (stratiform) on six orthogonal planes of variation. See Fig. 3 for more details.

observed along the $y$ axis $\left(0.575 \mathrm{~km} \mathrm{~min}^{-1}\right.$ for $Z_{\mathrm{h}}$ and $0.618 \mathrm{~km} \mathrm{~min}^{-1}$ for $Z_{\mathrm{dr}}$ ). By contrast, the apparent motion along the $x$ axis is more subtle $\left(0.035 \mathrm{~km} \mathrm{~min}^{-1}\right.$ for $Z_{\mathrm{h}}$ and $0.1 \mathrm{~km} \mathrm{~min}^{-1}$ for $\left.Z_{\mathrm{dr}}\right)$. The apparent motion along the vertical axis is close to zero for both $Z_{\mathrm{h}}$ and $Z_{\mathrm{dr}}$. The estimated time regularization factor is $1.72 \mathrm{~min} \mathrm{~km}^{-1}$ for $Z_{\mathrm{h}}$ and $1.56 \mathrm{~min} \mathrm{~km}^{-1}$ for $Z_{\mathrm{dr}}$, indicating a rather moderate temporal innovation.

Figure 5 shows the temporal variogram of $Z_{\mathrm{h}}$ obtained by considering the restriction of $\hat{\gamma}_{Z_{\mathrm{h}}}^{S T}$ to pure temporal variations (i.e., $\mathbf{h}=0$ ). The dotted line represents a fitted exponential model of the form

$$
\gamma_{Z_{\mathrm{h}}}^{T}(\tau)=n+(s-n)\left(1-e^{-3 \tau / r}\right)
$$

where $n$ is called the nugget, $s$ is the sill, and $r$ is the range. In this case, we have $n=8.26 \mathrm{dBZ} Z^{2}, s=$ $35.73 \mathrm{dBZ} Z^{2}$, and $r=63.54 \mathrm{~min}$. Using Eq. (23), we can scale $\hat{\gamma}_{Z_{\mathrm{h}}}^{T}$ according to $\mathbf{u}, \mathbf{A}$, and $\alpha$ to build a space-time variogram model, shown in Fig. 6 . The fact that the space-time model shown in Fig. 6 resembles the experimental variogram in Fig. 3 (at least close to the origin) corroborates the idea that the 3D-time correlation function of a rainfall field can indeed be estimated from its temporal correlation, provided that the motion, anisotropy, and time regularization factor of the field are known. Similarly good results are obtained for $Z_{\mathrm{dr}}$ (not shown here).

This ends the first part of the structural analysis. In the next part, we use the disdrometer data to infer the temporal structure of the DSD parameters and, from that, infer a 3D-time variogram model for $\mu$ and $N_{t}$.

\section{b. Temporal variogram of DSD parameters}

The disdrometer data consist of a collection of drop concentrations (at the ground level) at 30-s temporal resolution for 20 different diameter classes between 0.25

TABLE 1. Apparent motion $\mathbf{u}$, anisotropy $\mathbf{A}$, and time regularization factor $\alpha$ for event 1 (stratiform) and event 2 (convective).

\begin{tabular}{|c|c|c|c|c|}
\hline & \multicolumn{2}{|c|}{ Event 1} & \multicolumn{2}{|c|}{ Event 2} \\
\hline & $Z_{\mathrm{h}}$ & $Z_{\mathrm{dr}}$ & $Z_{\mathrm{h}}$ & $Z_{\mathrm{dr}}$ \\
\hline$u_{x}\left(\mathrm{~km} \mathrm{~min}^{-1}\right)$ & 0.035 & 0.1 & 0.67 & 0.65 \\
\hline$u_{y}\left(\mathrm{~km} \mathrm{~min}^{-1}\right)$ & 0.575 & 0.618 & -0.54 & -0.51 \\
\hline$u_{z}\left(\mathrm{~km} \mathrm{~min}^{-1}\right)$ & 0 & 0 & -0.37 & -0.33 \\
\hline$a(\mathrm{~km})$ & 1.0 & 1.0 & 0.65 & 0.67 \\
\hline$b(\mathrm{~km})$ & 0.29 & 0.35 & 0.39 & 0.36 \\
\hline$c(\mathrm{~km})$ & 0.89 & 0.90 & 1.0 & 1.0 \\
\hline$\theta_{x}(\mathrm{rad})$ & 1.59 & 1.54 & 2.34 & 2.26 \\
\hline$\theta_{y}(\mathrm{rad})$ & 2.56 & 2.67 & 0.77 & 0.81 \\
\hline$\theta_{z}(\mathrm{rad})$ & -0.09 & -0.12 & 0.39 & 0.38 \\
\hline$\alpha\left(\min \mathrm{km}^{-1}\right)$ & 1.72 & 1.56 & 0.61 & 0.66 \\
\hline
\end{tabular}




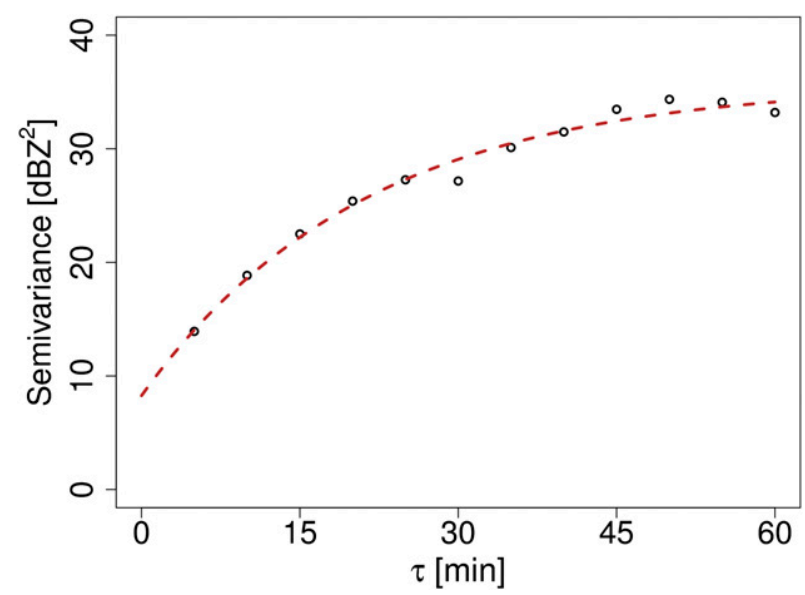

FIG. 5. Temporal variogram of $Z_{\mathrm{h}}$ with fitted exponential model.

and $7 \mathrm{~mm}$ and six different locations. All DSD spectra were manually processed, filtered, and quality controlled prior to analysis. For each disdrometer and each 30-s time step, the authors fitted a three-parameter Gamma DSD model using the maximum likelihood technique, taking into account the censoring of drop sizes below $0.25 \mathrm{~mm}$ of the Parsivel disdrometer. Figure 7 shows the time series of the fitted $\mu$ and $N_{t}$ values for the first disdrometer as well as the corresponding rain rate $R\left(\mathrm{~mm} \mathrm{~h}^{-1}\right)$ and the $\mu-\Lambda$ relationship. The time series of $\Lambda$ is not shown because it is highly correlated with $\mu$ (Spearman correlation coefficient of 0.94 ). The time series of the other disdrometers have similar characteristics and are not shown here.

A first visual inspection of the time series reveals that the fitted shape parameters $\mu$ are rather large. This corresponds to relatively narrow size distributions and large concentrations of small drops throughout the event, which is typical for stratiform rain. The concentration parameter $N_{t}$ is from low to moderate and weakly anticorrelated with $\mu$ (Spearman correlation coefficient of -0.13). The average drop size (not shown here) remains relatively low and constant over the course of the event. The rain rate is mostly influenced by variations in drop concentrations rather than in drop sizes, which is also typical for stratiform rain.

Figure 8 shows the temporal variograms of $\tilde{\mu}$ and $\tilde{N}_{t}$ for the first disdrometer, together with a fitted spherical model of the form

$$
\gamma(\tau)=n+(s-n)\left(\frac{3 \tau}{2 r}-\frac{\tau^{3}}{2 r^{3}}\right)
$$

The fitted model parameters are given in Table 2.

Figure 9 shows the estimated theoretical space-time variogram model of $\mu$ obtained by applying Eq. (23)
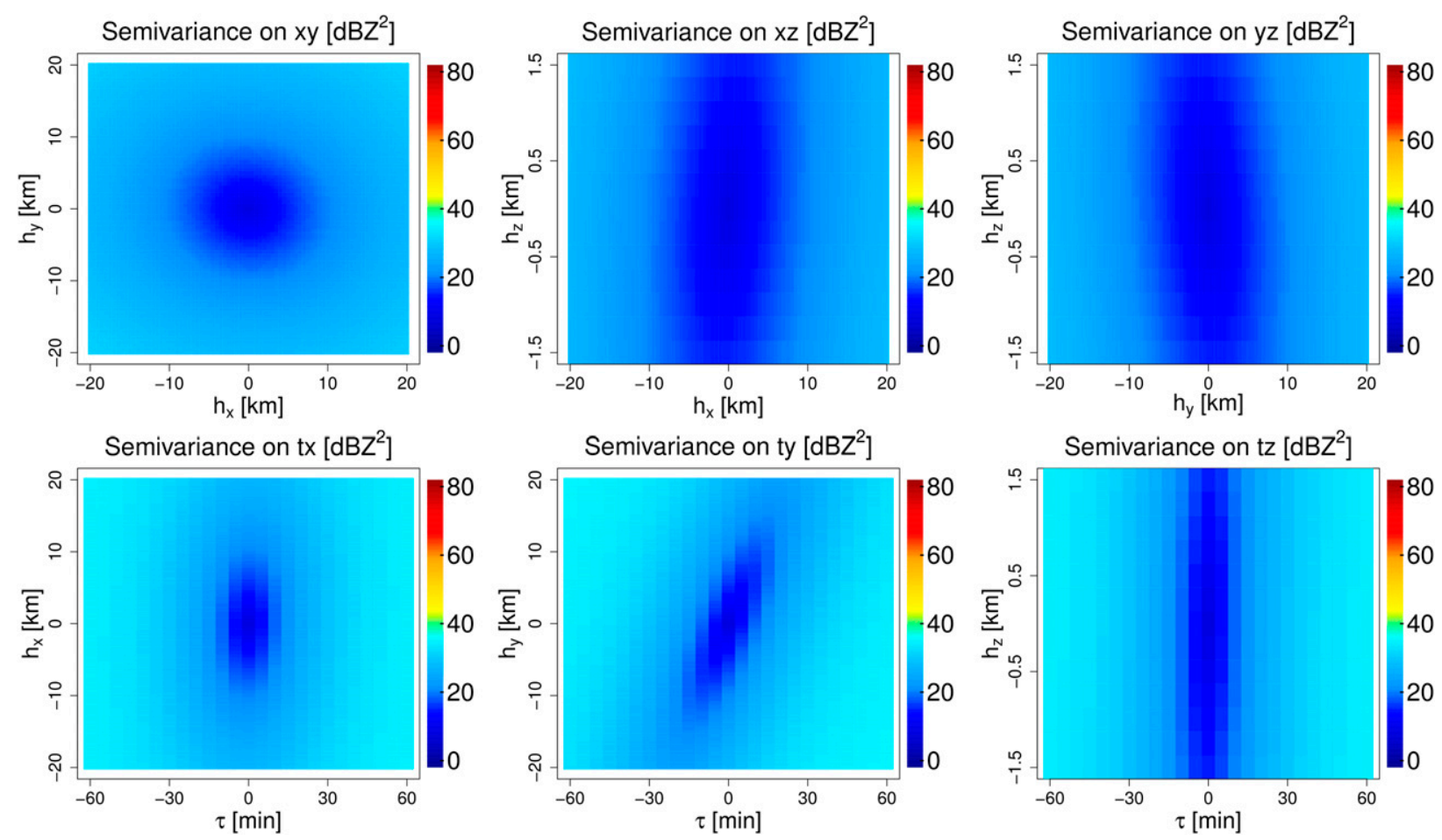

FIG. 6. Space-time variogram model for $Z_{\mathrm{h}}$ for event 1 (stratiform). This model was obtained by combining the temporal variogram in Fig. 5 with the sample estimates of $\mathbf{u}, \mathbf{A}$, and $\alpha$ in Table 1 . 

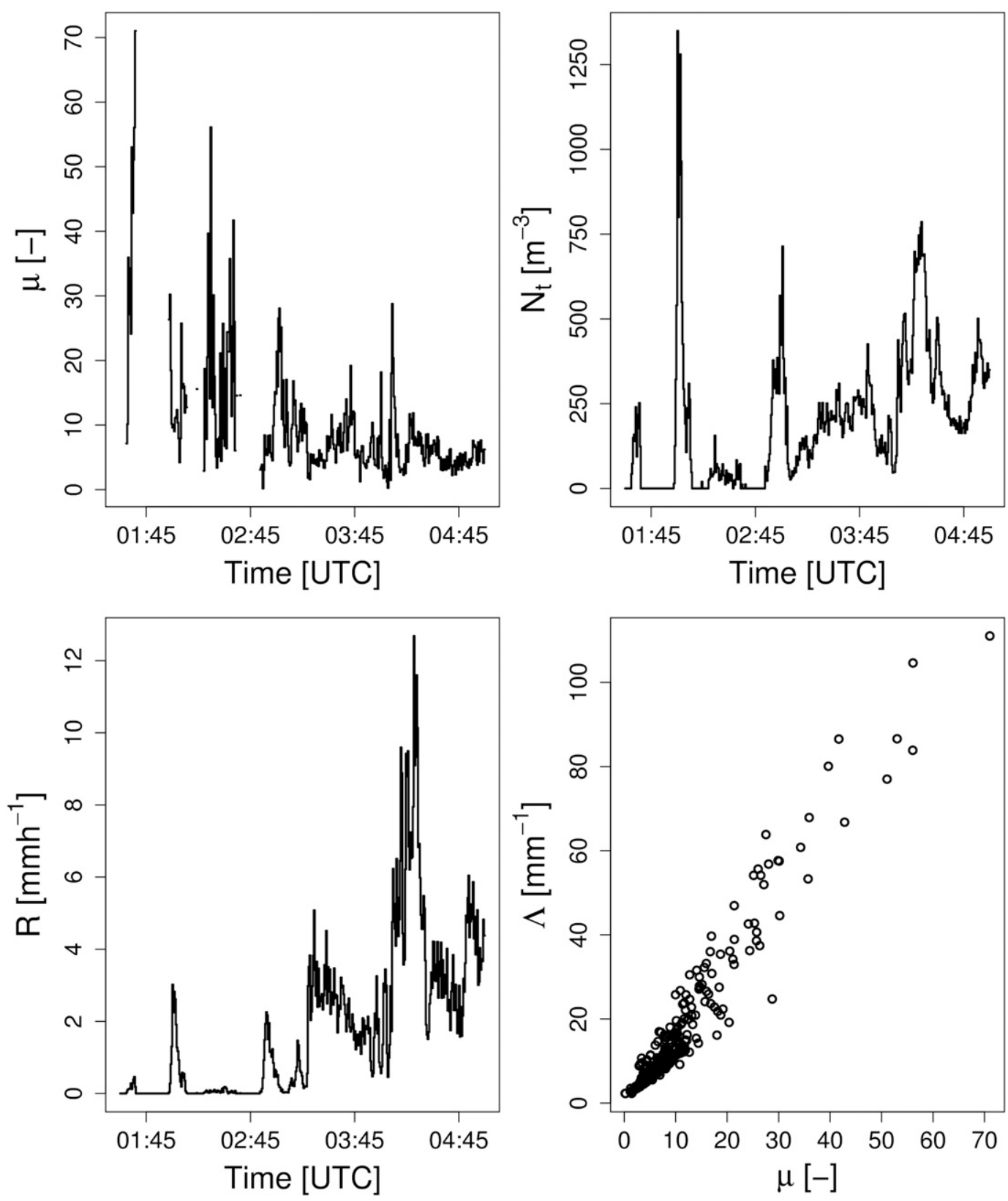

FIG. 7. Time series of $\mu$ (unitless), $N_{t}\left(\mathrm{~m}^{-3}\right), R\left(\mathrm{~mm} \mathrm{~h}^{-1}\right)$, and the $\mu-\Lambda$ relationship for event 1 (stratiform) and disdrometer 1 .

together with the estimated values of $\mathbf{u}, \mathbf{A}$, and $\alpha$ from $Z_{\mathrm{h}}$ (see previous section). The value of the scaling factor $\beta$ linking the temporal and space-time variograms is 0.86 . The same technique can be used to build a 3D-time variogram model for $N_{t}$ (not shown here).

\section{Case study 2: Convective event}

We consider a mesoscale convective system during 26-27 May 2013 over the state of Iowa during the Iowa
Flood Studies (IFloodS) experiment (http://gpm.nsstc. nasa.gov/ifloods/index.html). The system consists of numerous showers and thunderstorms, most of which initiated in the central plains at the nose of a low-level jet fueled by a constant feed of moisture coming from the south. As the system moves from west to east over the IFloodS domain, it is simultaneously observed by a dual-polarization S-band weather radar and 10 optical disdrometers of type Parsivel 2 (Tokay et al. 2014) at various locations in the domain. For homogeneity and 

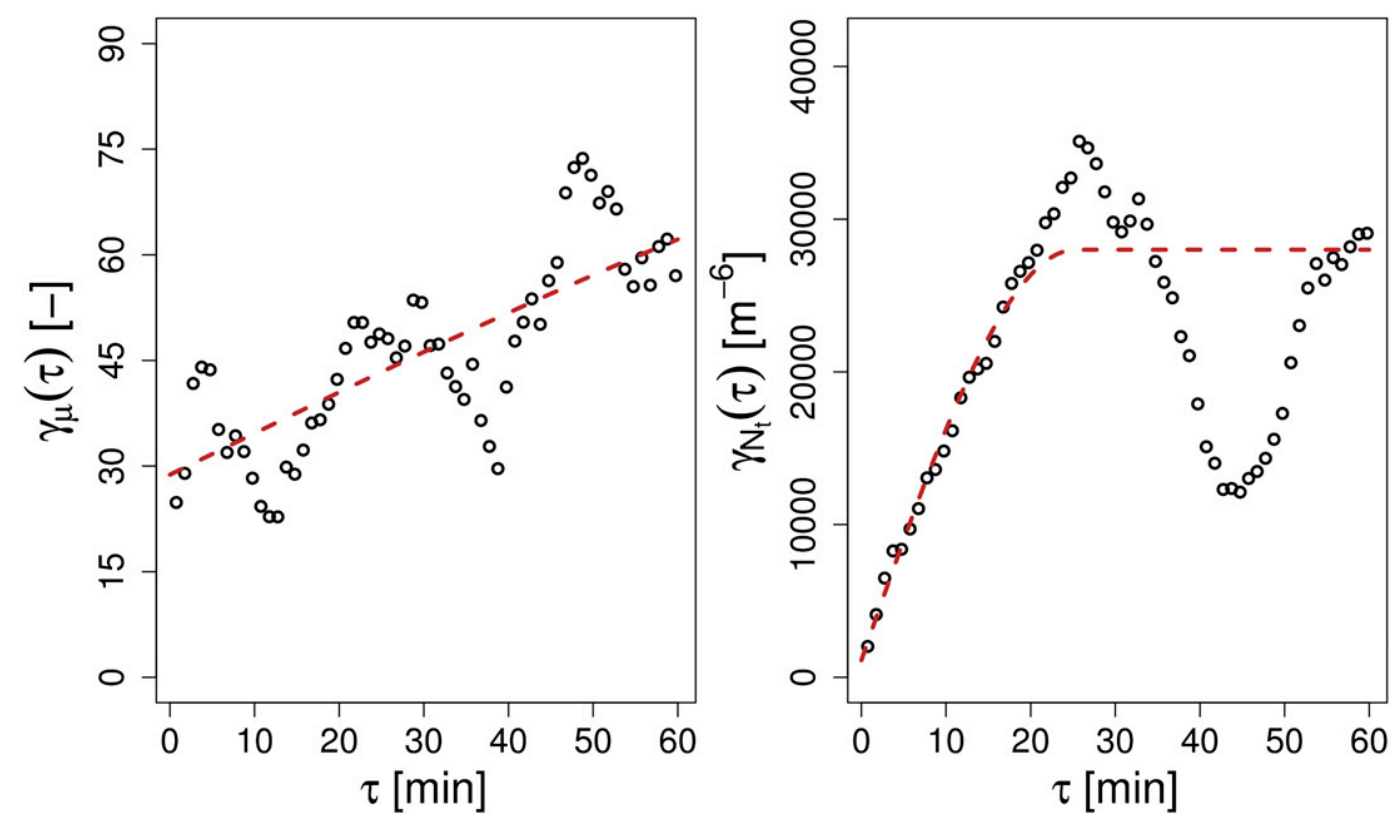

FIG. 8. Temporal variograms of $\mu$ and $N_{t}$ for event 1 (stratiform) and disdrometer 1 . The dotted lines represent the fitted spherical variogram models (see Table 2 for more details).

stationarity reasons, the analyses focus on a small area of size $100 \mathrm{~km} \times 100 \mathrm{~km} \times 2 \mathrm{~km}$ and a short time interval from 2030 to 0130 UTC during which the rainfall field was moving from northwest to southeast with approximately constant velocity. Animation 2 shows the temporal evolution of the radar reflectivity field (projected to the ground level) during this period (available as supplemental material at the Journals Online website: http://dx.doi.org/10.1175/JHM-D-14-0182.s2). Figure 10 shows two snapshots of the projected radar reflectivity field at 2300 and 2315 UTC together with the position of the Parsivel disdrometers in the area. One can recognize a large system of convective cells with extensive trailing stratiform precipitation in the northern and western parts of the domain. Figure 11 shows the local topography close to the radar (i.e., in the northwestern part of the domain depicted in Fig. 10).

Similar to the stratiform event, the case study consists of two main parts. First, the 3D-time radar reflectivity data are used to estimate the apparent motion, anisotropy, and time regularization factor. In the second part, we use the collected disdrometer data to estimate the temporal variogram of the DSD parameters and construct a theoretical 3D-time variogram model for each of the DSD parameters using Eq. (23).

\section{a. Space-time variogram of $Z_{\mathrm{h}}$ and $Z_{\mathrm{dr}}$}

The radar data used to derive the space-time variograms of $Z_{\mathrm{h}}$ and $Z_{\mathrm{dr}}$ consist of a large collection of
PPI and RHI scans of horizontal and vertical reflectivity $(\mathrm{dB} Z)$ at different elevation angles with a maximum range of $150 \mathrm{~km}$ and a range resolution of about $150 \mathrm{~m}$. To ensure only liquid precipitation is considered, the authors discarded all measurements above $2 \mathrm{~km}$ MSL. The remaining data were resampled over a Cartesian grid with $0.5-\mathrm{km}$ horizontal, $0.25-\mathrm{km}$ vertical, and 2.5-min temporal resolution and were used to compute the experimental space-time variogram of $Z_{\mathrm{h}}$ and $Z_{\mathrm{dr}}$ according to the procedure described in section 3. Given the resolution of the radar data, the variograms were computed using space-time classes of size $1.0 \mathrm{~km} \times 1.0 \mathrm{~km} \times 0.25 \mathrm{~km} \times 2.5 \mathrm{~min}$. To limit the computational cost, the spatial displacements were limited to a maximum of $20 \mathrm{~km}$ on the horizontal axes, $1.5 \mathrm{~km}$ on the vertical axis, and $60 \mathrm{~min}$ in time. The average number of samples per space-time class is about 6000 . Because of the radar scanning strategy, however, some space-time displacements are more

TABLE 2. Nugget $n$, sill $s$, and range $r$ of $\gamma_{\mu}^{T}$ and $\gamma_{N_{t}}^{T}$ for event 1 (stratiform) and event 2 (convective).

\begin{tabular}{cccccc}
\hline \hline & \multicolumn{2}{c}{ Event 1} & & \multicolumn{2}{c}{ Event 2} \\
\cline { 2 - 3 } \cline { 5 - 6 } & $\mu$ & $N_{t}$ & & $\mu$ & $N_{t}$ \\
\hline$n$ & 28.8 & $1115 \mathrm{~m}^{-6}$ & & 16.2 & $0 \mathrm{~m}^{-6}$ \\
$s$ & 86.9 & $28 \times 10^{3} \mathrm{~m}^{-6}$ & & 400 & $2 \times 10^{6} \mathrm{~m}^{-6}$ \\
$r$ & $148 \mathrm{~min}$ & $25.3 \mathrm{~min}$ & & $106.2 \mathrm{~min}$ & $50.3 \mathrm{~min}$ \\
\hline
\end{tabular}



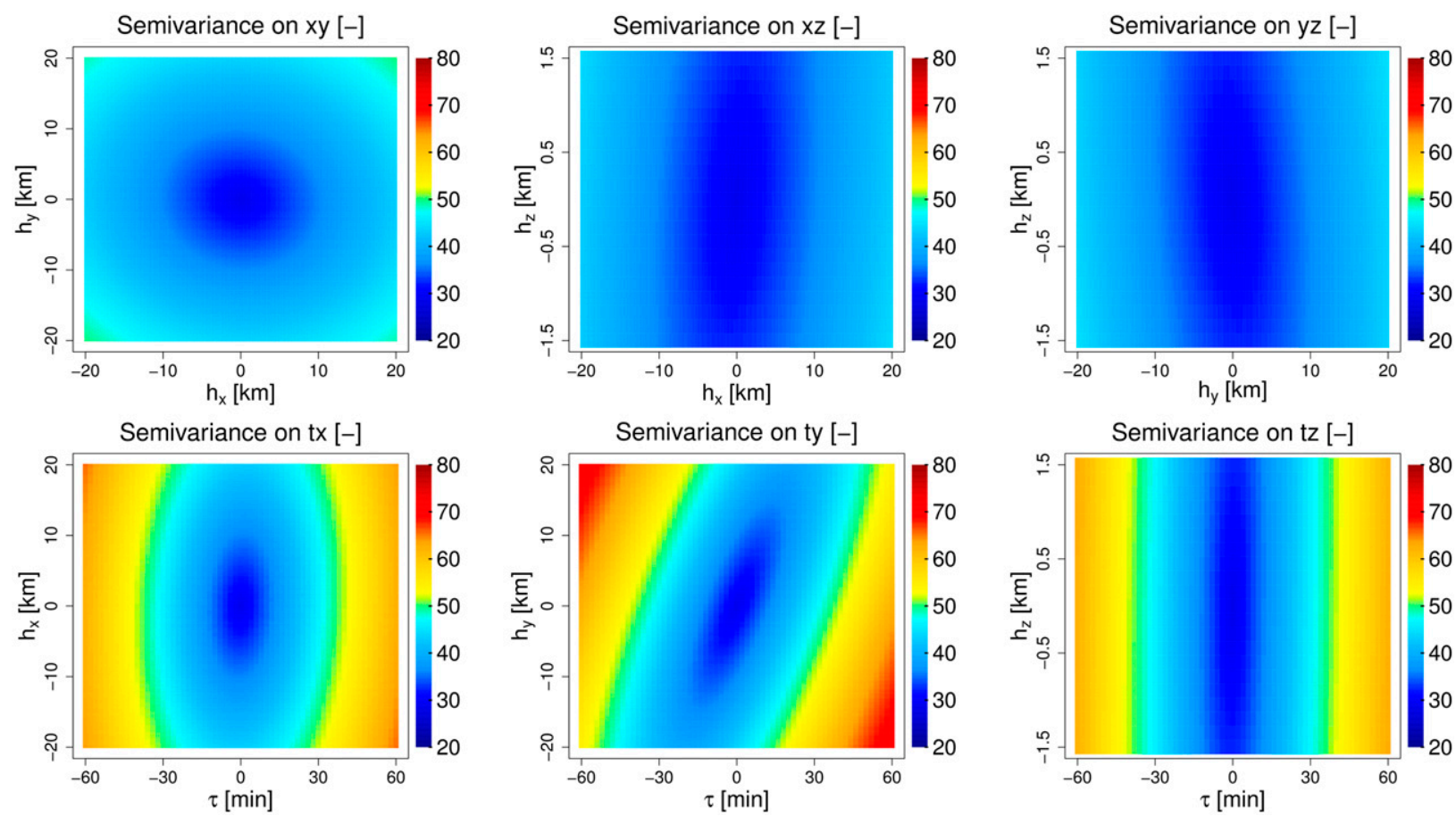

FIG. 9. Space-time variogram model for $\mu$ for event 1 (stratiform) and disdrometer 1 . This model was obtained by combining the temporal variogram of $\mu$ in Fig. 8 with the sample estimates of $\mathbf{u}, \mathbf{A}$, and $\alpha$ for $Z_{\mathrm{h}}$ (see Table 1 ).

common than others. To limit sampling issues, all space-time classes with fewer than 100 samples were discarded during the analysis.

Figures 12 and 13 show the obtained experimental space-time variograms of $Z_{\mathrm{h}}$ and $Z_{\mathrm{dr}}$. Similarly to event 1 , the shape and geometric characteristics of the two experimental space-time variograms are similar, meaning that the apparent motion $\mathbf{u}$, anisotropy $\mathbf{A}$, and time regularization $\alpha$ are the same no matter which moment of the DSD is considered. One can see the clear anisotropy of $Z_{\mathrm{h}}$ and $Z_{\mathrm{dr}}$ along all the three spatial planes of variations (see Table 1 for more details). The anisotropy along $x-y$ is consistent with the orientation of the convective cells in Fig. 10. The apparent motion of $Z_{\mathrm{h}}$
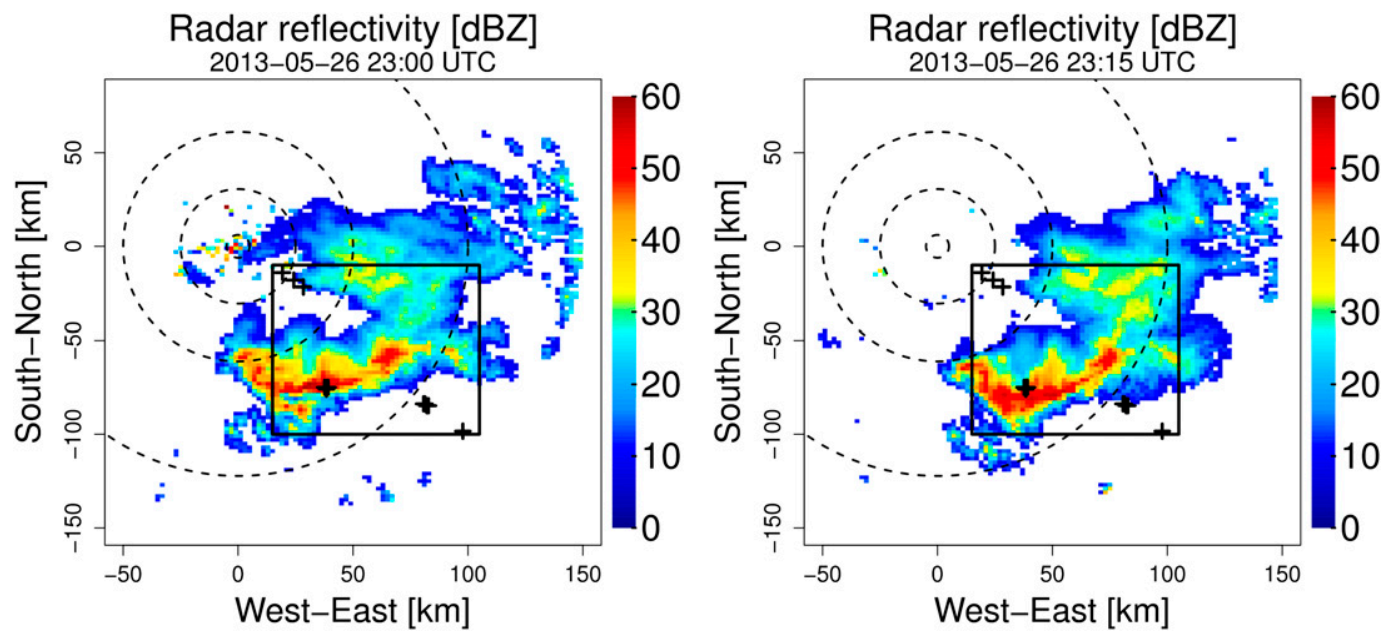

FIG. 10. Radar reflectivity maps (dBZ) for event 2 (convective) at (left) 2300 and (right) 2315 UTC, resampled over a Cartesian grid with $2 \times 2 \mathrm{~km}^{2}$ and 5-min resolution and projected to the ground level. The radar is located at the origin $(0,0)$ of the coordinate system. The concentric circles denote the 5-, 25-, 50-, and 100-km ranges. The locations of the Parsivel disdrometers are marked by black crosses. 


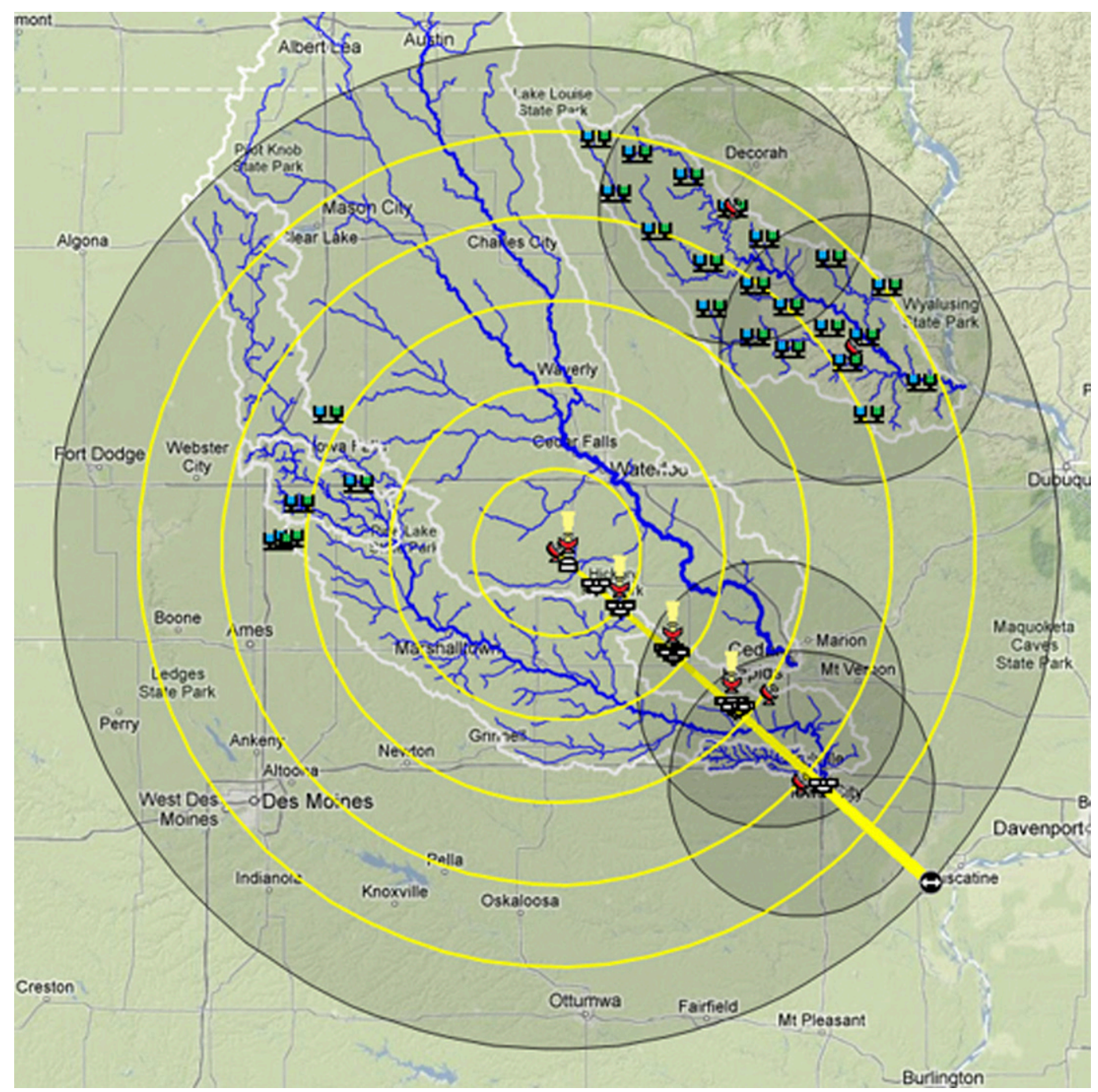

FIG. 11. Map for case study 2 (Iowa) with the location of the S-band weather radar (in the center) and some of the Parsivel disdrometers (in black). This map only shows the northwestern part of Fig. 10.

is estimated at $0.67 \mathrm{~km} \mathrm{~h}^{-1}$ along the $x$ axis $(0.65$ for $\left.Z_{\mathrm{dr}}\right),-0.54 \mathrm{~km} \mathrm{~h}^{-1}$ along the $y$ axis $\left(-0.51\right.$ for $\left.Z_{\mathrm{dr}}\right)$, and $-0.37 \mathrm{~km} \mathrm{~h}^{-1}$ along the $z$ axis $\left(-0.33\right.$ for $\left.Z_{\mathrm{dr}}\right)$. The time regularization factors are $0.61 \mathrm{~min} \mathrm{~km}^{-1}$ for $Z_{\mathrm{h}}$ and $0.66 \mathrm{~min} \mathrm{~km}^{-1}$ for $Z_{\mathrm{dr}}$, which means the fields are evolving more rapidly in time than for the stratiform case.

\section{b. Temporal variogram of DSD parameters}

The disdrometer data used to derive the temporal variograms of $\mu$ and $N_{t}$ consist of a collection of drop concentrations (at the ground level) at 1-min temporal resolution for 20 different diameter classes up to approximately $6 \mathrm{~mm}$ and 10 different locations. All DSD spectra were processed, filtered, and quality controlled prior to analysis. For each disdrometer and each 1-min time step, the authors fitted a three-parameter Gamma DSD model using the maximum likelihood technique. For conciseness, we only show the results for one of the 10 disdrometers in the southwest border of the study area (see Fig. 10), right under the convective part. The results for the remaining disdrometers are discussed at the end of the section. Figure 14 shows the time series of the fitted $\mu$ and $N_{t}$ values with associated rain rate $R$ and $\mu-\Lambda$ relationship for the selected disdrometer. The time series of $\Lambda$ (not shown) is highly correlated with $\mu$ (Spearman correlation coefficient of 0.98$)$.

In Fig. 14, one can see the large drop concentration values between up to 6000 drops per cubic meter together with some relatively low values of $\mu$ (i.e., large average drop sizes) around 2230 and before 0000 UTC. 

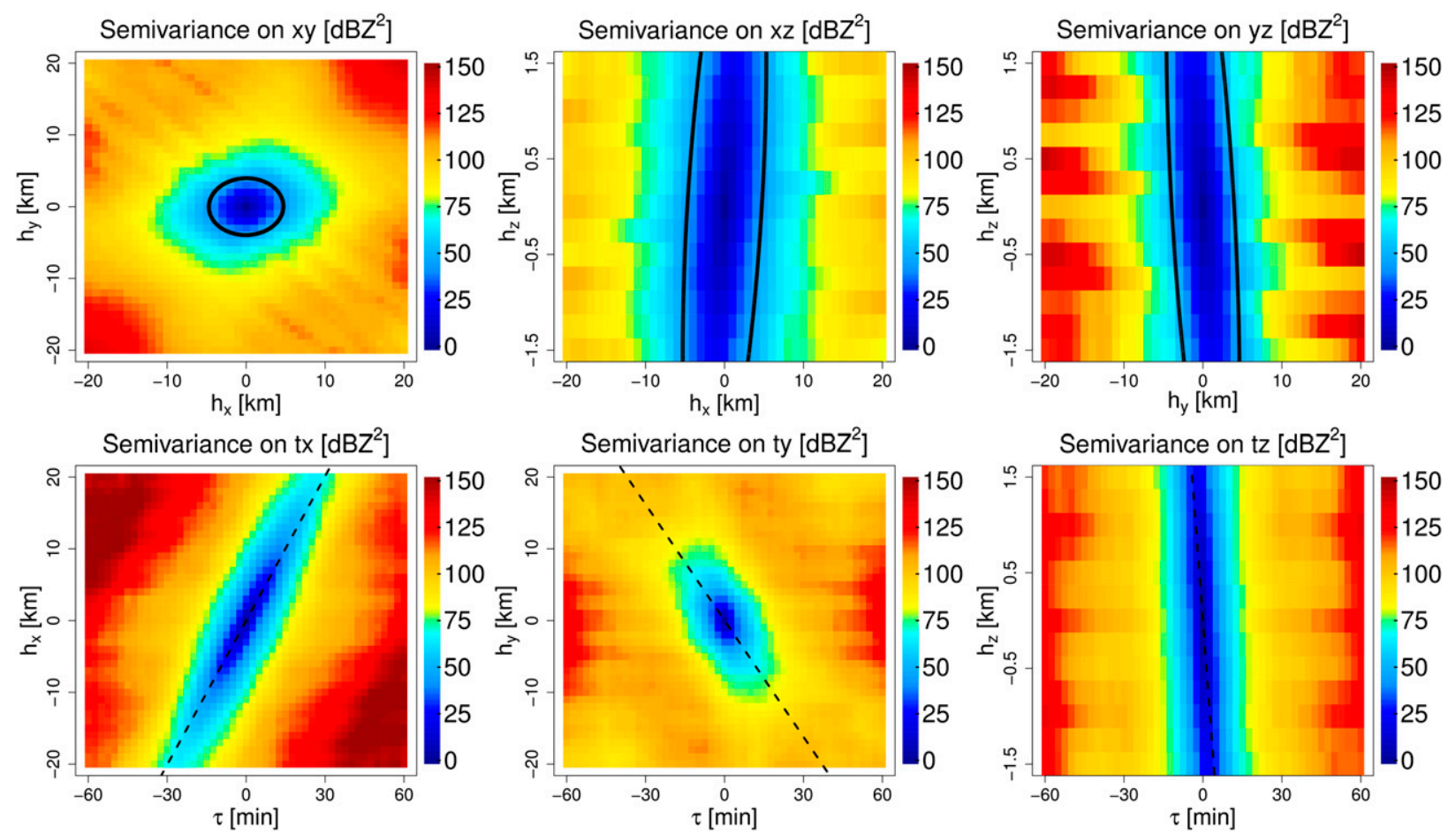

FIG. 12. Experimental space-time variogram of $Z_{\mathrm{h}}$ for event 2 (convective) on six orthogonal planes of variation. The class widths are $1.0 \mathrm{~km}$ for $h_{x}$ and $h_{y}, 0.25 \mathrm{~km}$ for $h_{z}$, and $2.5 \mathrm{~min}$ for $\tau$. The min number of samples per class is 100 . The ellipses represent the fitted anisotropy ellipsoid for a fixed value of semivariance. The dotted lines represent the fitted directions of min space-time variability along each axis.

These are typical values for convective events and are usually associated with higher rain rates. In contrast to the stratiform event, the average drop size (not shown here) fluctuates a lot over the course of the event. The rain rate is therefore influenced both by variations in drop concentrations and in drop sizes, with more rapid transitions than in the stratiform case. Similarly to the stratiform event, $N_{t}$ is weakly anticorrelated with $\mu$ (Spearman correlation coefficient of -0.21 ).

Figure 15 shows the temporal variograms of $\tilde{\mu}$ and $\tilde{N}_{t}$ for the selected disdrometer. The dotted lines represent the fitted spherical variogram model (see Table 2 for more details about the fitted nugget, sill, and range values). Note the large semivariance values of $\mu$ and $N_{t}$ compared with the stratiform event.

Figure 16 shows the estimated theoretical space-time variogram model of $N_{t}$ obtained by applying Eq. (23) together with the estimated values of $\mathbf{u}, \mathbf{A}$, and $\alpha$ from $Z_{\mathrm{h}}$. The 3D-time variogram model of $\mu$ (not shown) is obtained similarly. The value of the scaling factor $\beta$ linking the temporal and space-time variograms is 2.58 , which is much larger than for the stratiform event (0.86) and can be explained by the larger advection speed and the increased rate of temporal innovation (i.e., a smaller value of $\alpha$ ).

\section{c. Sensitivity to the choice of the disdrometer}

Note that the results above are based on the analysis of a single disdrometer located in the southwest border of the study area. To investigate the sensitivity of the method to the choice of the disdrometer, the authors repeated the same procedure for each of the remaining nine disdrometers in the domain. The results show that the retrieved space-time structures are strongly dependent on the choice of the disdrometer and their location with respect to the convective part of the precipitation field. Disdrometers close to each other are characterized by similar temporal variograms. There are, however, large differences between the northeast and the south of the domain. The reason for this is clear and can be explained by the location and movement of the convective cells shown in Fig. 10. From Animation 2 (available as supplemental material at the Journals Online website: http://dx.doi.org/10.1175/JHM-D-140182.s1), one can see that the disdrometers in the north of the domain completely missed the convective part of the event, leading to a different space-time structure than the rest of the disdrometers in the south. This highlights another important problem, which is heterogeneity and representativeness of data. It also means 

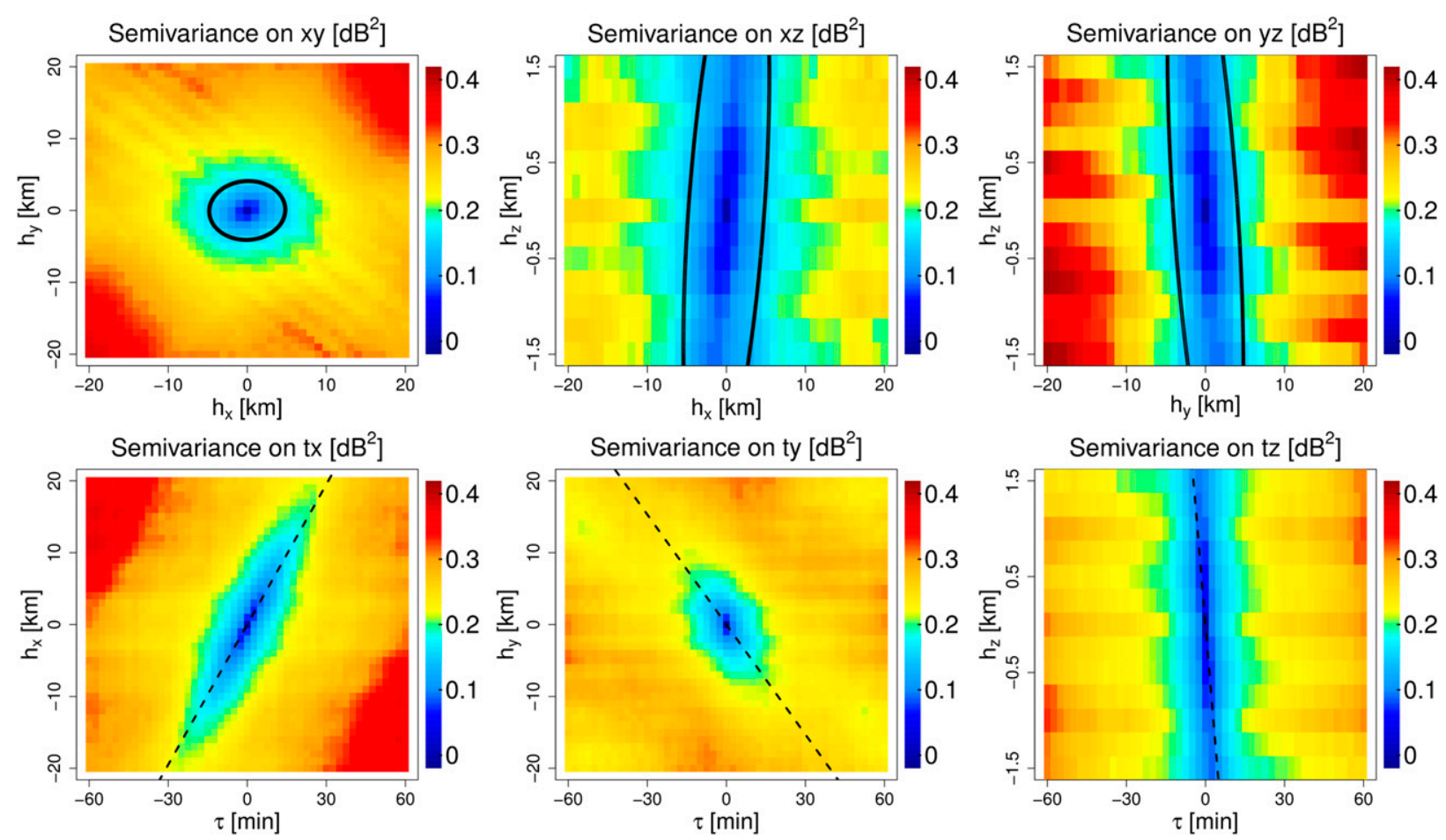

FIG. 13. Experimental space-time variogram of $Z_{\mathrm{dr}}$ for event 2 (convective) on six orthogonal planes of variation. See Fig. 12 for more details.

that the proposed method can only be applied to cases where the disdrometer(s) sees the "same" structures and dynamics as the radar.

\section{Discussion}

In the following, we shortly discuss the potential applications, limitations, and possible generalizations of the proposed geostatistical framework for analyzing the 3D-time variability of rainfall fields (including the DSD).

First, we can note that the proposed approach, independently of the possibility to retrieve the space-time structure of the DSD, is useful for quantifying and comparing the space-time structure of different rain events. It can therefore be used to build a climatology of rainfall variability and compare different rain events in terms of structure and dynamics rather than magnitude and location. A key parameter in this analysis is the time regularization factor $\alpha$, whose inverse describes how fast a rain event evolves in time. The two case studies in sections 4 and 5 suggest that $\alpha$ is smaller in convective rain, that is, the temporal innovation is faster for convective events. Of course, other cases need to be considered before any relevant conclusion can be drawn, but the interesting point here is that we have an objective method for comparing the rate at which the rainfall process evolves in time, independently of its geometry and of the advection process. Future studies will mainly focus on this issue and on the question of whether stratiform and convective elements in a given storm evolve at the same rate or not. Note that the methodology can also be applied to the output of numerical weather prediction models to compare the structure and dynamics of simulated events with radar observations. Additional insight can come from the analysis of the anisotropy matrix $\mathbf{A}$, which describes the relative variability along each spatial direction. Of particular interest are analyses that focus on the relative variability along the horizontal and vertical dimensions. This plays an important role in radar remote sensing applications and could be used to assess the uncertainty associated with projecting radar measurements to the ground.

The second interesting application of the model proposed in this paper is rainfall interpolation and stochastic simulation. The space-time variogram model of $Z_{\mathrm{h}}$ can be used to krige radar reflectivity fields in space and time (e.g., Delhomme 1978; Isaaks and Srivastava 1989; Goovaerts 1997; Chilès and Delfiner 1999) and to investigate the uncertainty associated with radar nowcasts. The 3D-time variogram models of the DSD 

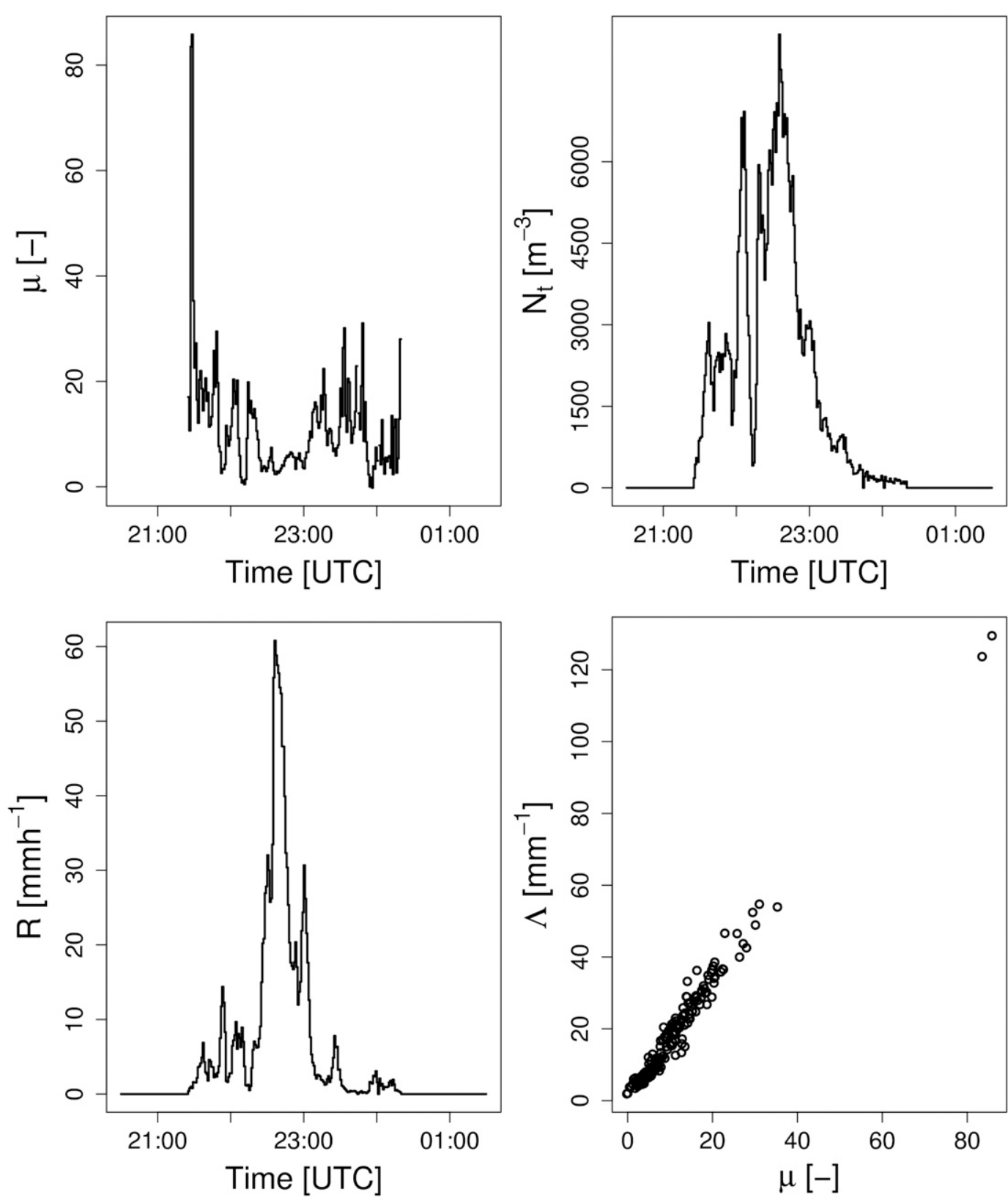

FIG. 14. Time series of $\mu$ (unitless), $N_{t}\left(\mathrm{~m}^{-3}\right), R\left(\mathrm{~mm} \mathrm{~h}^{-1}\right)$, and the $\mu-\Lambda$ relationship for event 2 (convective) and disdrometer 7 .

parameters $\mu$ and $N_{t}$ can be used to perform conditional and unconditional stochastic simulation of synthetic high-resolution DSD fields with prescribed space-time correlation structure (e.g., Lanza 2000; Schleiss et al. 2009, 2012; Leblois and Creutin 2013; Paschalis et al. 2013). These 3D-time fields can be used as a reference to test and assess the performance of various remote sensing algorithms like attenuation correction and optimum combination of measurements from several overlapping radars.
One of the main limitations of the methodology proposed in this paper is the fact that the apparent motion $\mathbf{u}$ and time regularization factor $\alpha$ must be assumed constant over the course of the event. While this might be reasonable over relatively small domains and short time intervals, it inevitably breaks down at larger scales. In the following, we briefly discuss some alternative for the case where $\mathbf{u}$ is a function of location $\mathbf{x}$ and time $t$. The easiest way, of course, is to separate the domain into several parts and perform the structural analysis for each 

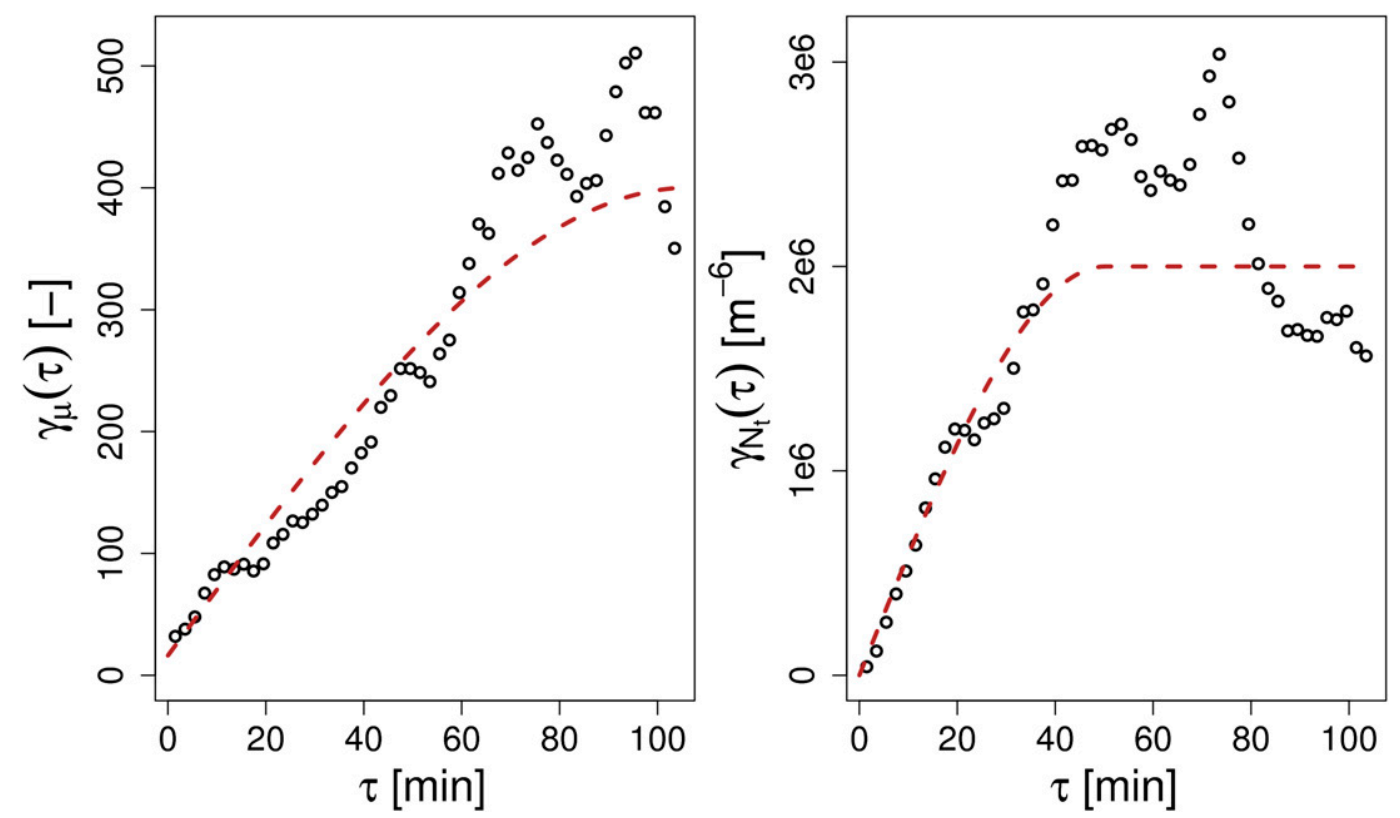

FIG. 15. Temporal variograms of $\mu$ and $N_{t}$ for event 2 (convective) and selected disdrometer. The dotted lines represent the fitted spherical variogram models (see Table 2 for more details).

part separately. The alternative approach is to consider a single large domain and to extend the proposed methodology to variable motion fields. The formalism becomes more complicated but the basic idea remains the same: estimate the local motion field $\mathbf{u}(\mathbf{x}, t)$ using a cell/echo tracking technique and use it to define an appropriate Lagrangian coordinate system in which the spatial structure of the rainfall process can be analyzed independently of its motion. More specifically, we use the concept of back trajectory to compute the position of any space-time point $(\mathbf{x}, t)$ at time $t=0$ :

$$
(\mathbf{x}, t) \mapsto\left(\mathbf{A x}^{\prime}, \frac{t}{\alpha}\right)
$$

where $\mathbf{x}^{\prime}$ denotes the location of $(\mathbf{x}, t)$ back projected at $t=0$ using $\mathbf{u}(\mathbf{x}, t)$. Similarly, one can define a temporally dependent time regularization factor $\alpha(t)$ :

$$
(\mathbf{x}, t) \mapsto\left(\mathbf{A} \mathbf{x}^{\prime}, t^{\prime}\right),
$$

where $t^{\prime}=\int_{0}^{t} \alpha(s) d s$. The major problem when considering a nonuniform motion field or a time-dependent regularization factor is the fact that $\mathbf{u}$ and $\alpha$ might not be well defined everywhere along the back trajectory. This makes it difficult to compute $\mathbf{x}^{\prime}$ and to estimate the associated space-time variogram. It also makes the scaling factor $\beta$ in Eq. (21) dependent on the location at which the time series was measured.

Another important point is rainfall intermittency and how it affects the space-time structure of the rainfall process. This is a difficult problem that has been ignored in this paper but will have to be investigated more thoroughly in future studies. A possible approach for dealing with intermittent fields would be to use the recently introduced concept of "dry drift" (Schleiss et al. 2014). One could, for example, use the same space-time metric $\delta$ in Eq. (9) to define the space-time variogram and the dry drift. The question that remains, however, is how well the dry drift can be estimated in practice knowing that the considered radar reflectivity fields usually have a limited vertical extent (i.e., a few kilometers from the ground level up to the melting layer). This is beyond the scope of this paper and will have to be addressed in future studies.

\section{Conclusions}

Rainfall is a complex dynamic system covering many scales in space and time. Many state-of-the-art remote sensing and hydrological applications require precise knowledge of the space-time correlation structure and temporal evolution of rainfall. In this paper, we proposed a geostatistical method for quantifying the 3D-time structure of rainfall fields (including the DSD) from the ground level up to the melting layer using available radar and disdrometer data. The method was successfully applied to two different events in southern France and the midwestern United States with promising results. 

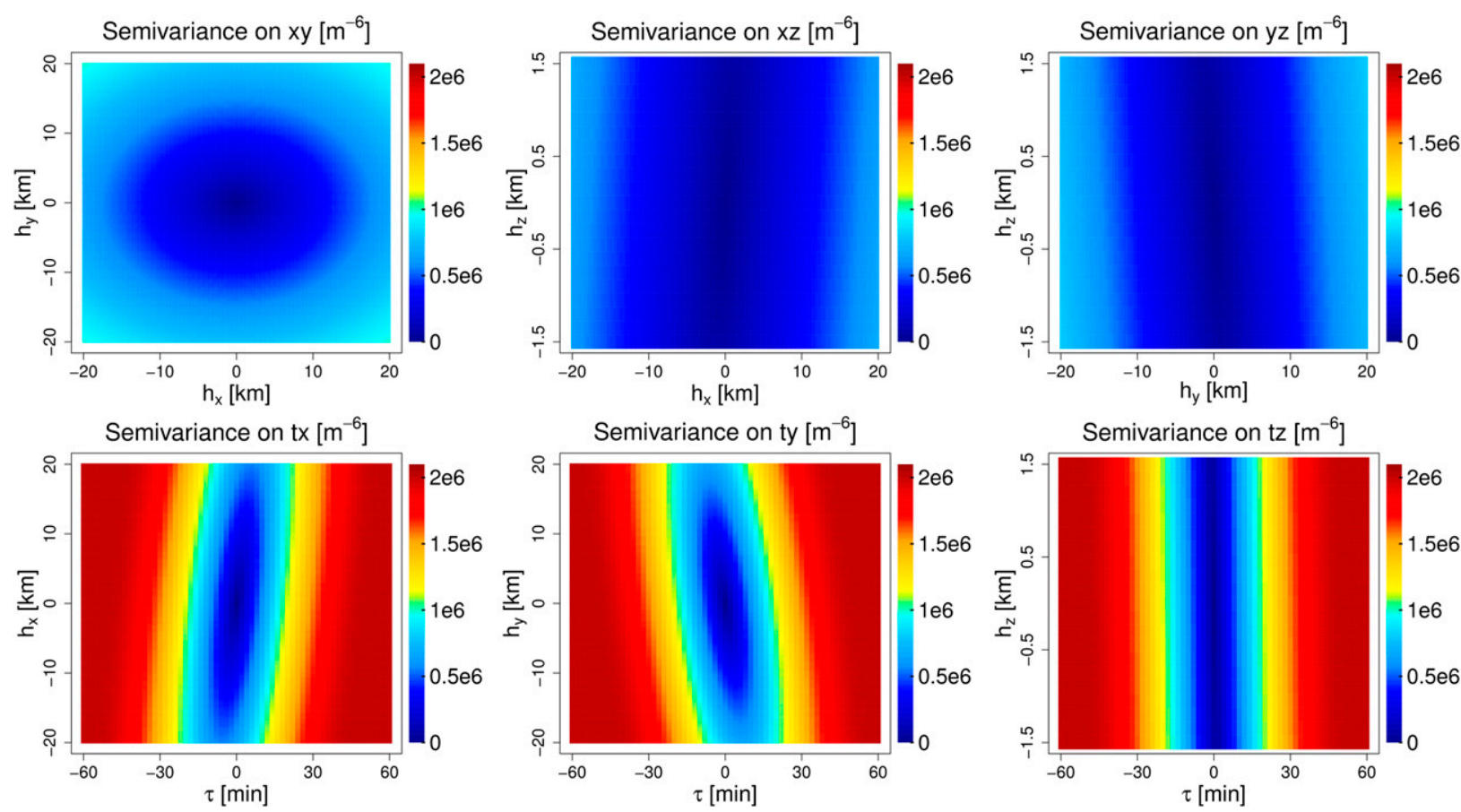

FIG. 16. Space-time variogram model for $N_{t}$ for event 2 (convective) and selected disdrometer. This model was obtained by combining the temporal variogram of $N_{t}$ in Fig. 15 with the sample estimates of $\mathbf{u}, \mathbf{A}$, and $\alpha$ for $Z_{\mathrm{h}}$ (see Table 1).

A key point in the proposed approach is the theoretical link between the space-time variability of a moving rainfall field and the temporal variability observed at the ground level. More specifically, we show that the spacetime variogram of any DSD-related quantity can be expressed as a scaled version of its temporal variogram. The conversion from time to space-time is performed using a generalization of Taylor's hypothesis of frozen turbulence, which takes into account the apparent motion, anisotropy, and time regularization factor of the rainfall process. The result is a simplified and idealized statistical representation of rainfall variability that is wrong from the physical point of view but has the merit of making the best out the available data. It also offers an intuitive framework for analyzing and comparing rainfall structures.

Acknowledgments. This work is a contribution to the HyMeX program and IFloodS campaign through project STORM (Spatial and Temporal Variability of Rainfall Microstructure) funded by Grant P2ELP2 148878 of the Swiss National Science Foundation. The authors thank Alexis Berne, Jacopo Grazioli, and Timothy Raupach from EPFL-LTE for collecting and providing the radar and disdrometer data used in the first case study. The authors also acknowledge the NASA GPM Ground Validation Program, the Iowa Flood Center, and especially those in the NASA Wallops
Flight Facility GV group for their efforts in collection of the field data used in the second case study.

\section{REFERENCES}

Barthes, L., and C. Mallet, 2013: Vertical evolution of raindrop size distribution: Impact on the shape of the DSD. Atmos. Res., 119, 13-22, doi:10.1016/j.atmosres.2011.07.011.

Bowler, N. E. H., C. E. Pierce, and A. Seed, 2004: Development of a precipitation nowcasting algorithm based upon optical flow techniques. J. Hydrol., 288, 74-91, doi:10.1016/ j.jhydrol.2003.11.011.

Brandes, E. A., G. Zhang, and J. Vivekanandan, 2004: Drop size distribution retrieval with polarimetric radar: Model and application. J. Appl. Meteor., 43, 461-475, doi:10.1175/ 1520-0450(2004)043<0461:DSDRWP>2.0.CO;2.

Bringi, V. N., V. Chandrasekar, J. Hubbert, E. Gorgucci, W. L. Randeu, and M. Schoenhuber, 2003: Raindrop size distribution in different climatic regimes from disdrometer and dualpolarized radar analysis. J. Atmos. Sci., 60, 354-365, doi:10.1175/1520-0469(2003)060<0354:RSDIDC >2.0.CO;2.

Chilès, J.-P., and P. Delfiner, 1999: Geostatistics: Modeling Spatial Uncertainty. Wiley, $695 \mathrm{pp}$.

Cifelli, R., W. A. Petersen, L. D. Carey, S. A. Rutledge, and M. A. F. D. Dias, 2002: Radar observations of the kinematic, microphysical, and precipitation characteristics of two MCSs in TRMM LBA. J. Geophys. Res., 107, 8077, doi:10.1029/2000JD000264.

Crane, R. K., 1979: Automatic cell detection and tracking. IEEE Trans. Geosci. Electron., 17, 250-262, doi:10.1109/ TGE.1979.294654.

Delhomme, J.-P., 1978: Kriging in the hydrosciences. Adv. Water Resour., 1, 251-266, doi:10.1016/0309-1708(78)90039-8. 
Dixon, M., and G. Wiener, 1993: TITAN: Thunderstorm Identification, Tracking, Analysis, and Nowcasting-A radar-based methodology. J. Atmos. Oceanic Technol., 10, 785-797, doi:10.1175/1520-0426(1993)010<0785:TTITAA > 2.0.CO;2.

Ducrocq, V., and Coauthors, 2014: HyMeX-SOP1: The field campaign dedicated to heavy precipitation and flash flooding in the northwestern Mediterranean. Bull. Amer. Meteor. Soc., 95, 1083-1100, doi:10.1175/BAMS-D-12-00244.1.

Gneiting, T., 2002: Nonseparable, stationary covariance functions for space-time data. J. Amer. Stat. Assoc., 97, 590-600, doi:10.1198/016214502760047113.

Goovaerts, P., 1997: Geostatistics for Natural Resources Evaluation. Oxford University Press, 483 pp.

Gorgucci, E., V. Chandrasekar, V. N. Bringi, and G. Scarchilli, 2002: Estimation of raindrop size distribution parameters from polarimetric radar measurements. J. Atmos. Sci., 59, 2373-2384, doi:10.1175/1520-0469(2002)059<2373:EORSDP>2.0.CO;2.

Hou, A. Y., and Coauthors, 2014: The Global Precipitation Measurement mission. Bull. Amer. Meteor. Soc., 95, 701-722, doi:10.1175/BAMS-D-13-00164.1.

Isaaks, E. H., and R. M. Srivastava, 1989: An Introduction to Applied Geostatistics. Oxford University Press, $561 \mathrm{pp}$.

Jaffrain, J., A. Studzinski, and A. Berne, 2011: A network of disdrometers to quantify the small-scale variability of the raindrop size distribution. Water Resour. Res., 47, W00H06, doi:10.1029/2010WR009872.

Joss, J., and E. Gori, 1978: Shapes of raindrop size distributions. J. Appl. Meteor., 17, 1054-1061, doi:10.1175/1520-0450(1978)017<1054: SORSD $>2.0 . \mathrm{CO} ; 2$.

Kim, D. S., M. Maki, and D. I. Lee, 2010: Retrieval of threedimensional raindrop size distribution using X-band polarimetric radar data. J. Atmos. Oceanic Technol., 27, 1265-1285, doi:10.1175/2010JTECHA1407.1.

Kozu, T., K. K. Reddy, S. Mori, M. Thurai, J. T. Ong, D. N. Rao, and T. Shimomai, 2006: Seasonal and diurnal variations of raindrop size distribution in Asian monsoon region. J. Meteor. Soc. Japan, 84A, 195-209, doi:10.2151/jmsj.84A.195.

Lanza, L., 2000: A conditional simulation model of intermittent rain fields. Hydrol. Earth Syst. Sci., 4, 173-183, doi:10.5194/ hess-4-173-2000.

Le, M., and V. Chandrasekar, 2014: An algorithm for drop-size distribution retrieval from GPM dual-frequency precipitation radar. IEEE Trans. Geosci. Remote Sens., 52, 7170-7185, doi:10.1109/TGRS.2014.2308475.

Leblois, E., and J. D. Creutin, 2013: Space-time simulation of intermittent rainfall with prescribed advection field: Adaptation of the turning band method. Water Resour. Res., 49, 33753387, doi:10.1002/wrcr.20190.

Lepioufle, J.-M., E. Leblois, and J.-D. Creutin, 2012: Variography of rainfall accumulation in presence of advection. J. Hydrol., 464-465, 494-504, doi:10.1016/j.jhydrol.2012.07.041.

Li, B., A. Murthi, K. Bowman, G. North, M. Genton, and M. Sherman, 2009: Statistical tests of Taylor's hypothesis: An application to precipitation fields. J. Hydrometeor., 10, 254 265, doi:10.1175/2008JHM1009.1.

Liao, L., R. Meneghini, and A. Tokay, 2014: Uncertainties of GPM DPR rain estimates caused by DSD parameterizations. J. Appl. Meteor. Climatol., 53, 2524-2537, doi:10.1175/ JAMC-D-14-0003.1.

Löffler-Mang, M., and J. Joss, 2000: An optical disdrometer for measuring size and velocity of hydrometeors. J. Atmos. Oceanic Technol., 17, 130-139, doi:10.1175/1520-0426(2000)017<0130: AODFMS $>2.0 . \mathrm{CO} ; 2$.
Marshall, J. S., and W. M. Palmer, 1948: The distribution of raindrops with size. J. Meteor., 5, 165-166, doi:10.1175/1520-0469 (1948)005<0165:TDORWS >2.0.CO;2.

Mecklenburg, S., J. Joss, and W. Schmid, 2000: Improving the nowcasting of precipitation in an Alpine region with an enhanced radar echo tracking algorithm. J. Hydrol., 239, 46-68, doi:10.1016/S0022-1694(00)00352-8.

Onof, C., P. Northrop, H. S. Wheater, and V. Isham, 1996: Spatiotemporal storm structure and scaling property analysis for modeling. J. Geophys. Res., 101, 26415-26 425, doi:10.1029/ 96JD01378.

Paschalis, A., P. Molnar, S. Fatichi, and P. Burlando, 2013: A stochastic model for high-resolution space-time precipitation simulation. Water Resour. Res., 49, 8400-8417, doi:10.1002/ 2013WR014437.

Peters, G., B. Fischer, and T. Andersson, 2002: Rain observations with a vertically looking Micro Rain Radar (MRR). Boreal Environ. Res., 7 (4), 353-362.

,-- H. Munster, M. Clemens, and A. Wagner, 2005: Profiles of raindrop size distributions as retrieved by microrain radars. J. Appl. Meteor., 44, 1930-1949, doi:10.1175/ JAM2316.1.

Potvin, G., 1993: Space-time correlations and Taylor's hypothesis for rainfall. M.S. thesis, Department of Physics, McGill University, $68 \mathrm{pp}$.

Schleiss, M., A. Berne, and R. Uijlenhoet, 2009: Geostatistical simulation of two-dimensional fields of raindrop size distributions at the meso- $\gamma$ scale. Water Resour. Res., 45, W07415, doi:10.1029/2008WR007545.

- J. Jaffrain, and A. Berne, 2012: Stochastic simulation of intermittent DSD fields in time. J. Hydrometeor., 13, 621-637, doi:10.1175/JHM-D-11-018.1.

— S. Chamoun, and A. Berne, 2014: Nonstationarity in intermittent rainfall: The "dry drift." J. Hydrometeor., 15, 11891204, doi:10.1175/JHM-D-13-095.1.

Seifert, A., 2005: On the shape-slope relation of drop size distributions in convective rain. J. Appl. Meteor., 44, 1146-1151, doi:10.1175/JAM2254.1.

Sempere Torres, D., J. M. Porrà, and J.-D. Creutin, 1994: A general formulation for raindrop size distribution. J. Appl. Meteor., 33, 1494-1502, doi:10.1175/1520-0450(1994)033<1494: AGFFRS $>2.0 . \mathrm{CO} ; 2$.

Smith, E., and Coauthors, 2007: International Global Precipitation Measurement (GPM) program and mission: An overview. Measuring Precipitation From Space, V. Levizzani, P. Bauer, and F. J. Turk, Eds., Advances in Global Change Research, Vol. 28, Springer, 611-653, doi:10.1007/978-1-4020-5835-6_48.

Smith, J. A., E. Hui, M. Steiner, M. L. Baeck, W. Krajewski, and A. A. Ntelekos, 2009: Variability of rainfall rate and raindrop size distributions in heavy rain. Water Resour. Res., 45, W04430, doi:10.1029/2008WR006840.

Tapiador, F. J., R. Checa, and M. de Castro, 2010: An experiment to measure the spatial variability of rain drop size distribution using sixteen laser disdrometers. Geophys. Res. Lett., 37, L16803, doi:10.1029/2010GL044120.

Taylor, G. I., 1938: The spectrum of turbulence. Proc. Roy. Soc. London, 164, 476-490, doi:10.1098/rspa.1938.0032.

Tokay, A., D. B. Wolff, and W. A. Petersen, 2014: Evaluation of the new version of the laser-optical disdrometer, OTT Parsivel2. J. Atmos. Oceanic Technol., 31, 1276-1288, doi:10.1175/ JTECH-D-13-00174.1.

Uijlenhoet, R., and J. H. Pomeroy, 2001: Raindrop size distributions and radar reflectivity-rain rate relationships for radar 
hydrology. Hydrol. Earth Syst. Sci., 5, 615-628, doi:10.5194/ hess-5-615-2001.

Ulbrich, C. W., 1983: Natural variations in the analytical form of the raindrop size distribution. J. Climate Appl. Meteor., 22, 1764-1775, doi:10.1175/1520-0450(1983)022<1764: NVITAF $>2.0 . \mathrm{CO} ; 2$.

, and D. Atlas, 2007: Microphysics of raindrop size spectra: Tropical continental and maritime storms. J. Appl. Meteor. Climatol., 46, 1777-1791, doi:10.1175/2007JAMC1649.1.

Williams, C. R., 2002: Simultaneous ambient air motion and raindrop size distributions retrieved from UHF vertical incident profiler observations. Radio Sci., 37, 1024, doi:10.1029/ 2000RS002603.

- and Coauthors, 2014: Describing the shape of raindrop size distributions using uncorrelated raindrop mass spectrum parameters. J. Appl. Meteor. Climatol., 53, 1282-1296, doi:10.1175/JAMC-D-13-076.1.

Willis, P. T., 1984: Functional fits to some observed drop size distributions and parameterization of rain. J. Atmos. Sci.,
41, 1648-1661, doi:10.1175/1520-0469(1984)041<1648: FFTSOD $>2.0 . \mathrm{CO} ; 2$.

Yoshikawa, E., S. Kida, S. Yoshida, T. Morimoto, T. Ushio, and Z. Kawasaki, 2010: Vertical structure of raindrop size distribution in lower atmospheric boundary layer. Geophys. Res. Lett., 37, L20802, doi:10.1029/2010GL045174.

Yuter, S. E., and R. A. J. Houze, 2003: Microphysical modes of precipitation growth determined by S-band vertically pointing radar in orographic precipitation during MAP. Quart. J. Roy. Meteor. Soc., 129, 455-476, doi:10.1256/qj.01.216.

Zhang, G., J. Vivekanandan, and E. Brandes, 2001: A method for estimating rain rate and drop size distribution from polarimetric radar measurements. IEEE Trans. Geosci. Remote Sens., 39, 830-841, doi:10.1109/36.917906.

- —_, _ - R. Meneghini, and T. Kozu, 2003: The shape-slope relation in observed gamma raindrop size distributions: Statistical error or useful information? J. Atmos. Oceanic Technol., 20, 1106-1119, doi:10.1175/1520-0426(2003)020<1106: TSRIOG $>2.0 . \mathrm{CO} ; 2$. 